\title{
Development and Use of Engineering Standards for Computational Fluid Dynamics for Complex Aerospace Systems
}

\author{
Hyung B. Lee \\ Bettis Laboratory, West Mifflin, Pennsylvania, United States \\ Urmila Ghia \\ University of Cincinnati, Cincinnati, Ohio, United States \\ Sami Bayyuk \\ ESI Group US R\&D, Huntsville, Alabama, United States \\ William L. Oberkampf \\ WLO Consulting, Austin, Texas, United States \\ Christopher J. Roy \\ Virginia Tech, Blacksburg, Virginia, United States \\ John A. Benek \\ Air Force Research Laboratory, Wright-Patterson AFB, Ohio, United States \\ Christopher L. Rumsey \\ NASA Langley Research Center, Hampton, Virginia, United States \\ Joseph M. Powers \\ University of Notre Dame, Notre Dame, Indiana, United States \\ Robert H. Bush \\ Pratt and Whitney, East Hartford, Connecticut, United States \\ Mortaza Mani \\ Boeing Research and Technology, St. Louis, Missouri, United States
}

\begin{abstract}
Computational fluid dynamics (CFD) and other advanced modeling and simulation (M\&S) methods are increasingly relied on for predictive performance, reliability and safety of engineering systems. Analysts, designers, decision makers, and project managers, who must depend on simulation, need practical techniques and methods for assessing simulation credibility. The AIAA Guide for Verification and Validation of Computational Fluid Dynamics Simulations (AIAA G-077-1998 (2002)), originally published in 1998, was the first engineering standards document available to the engineering community for verification and validation (V\&V) of simulations. Much progress has been made in these areas since 1998. The AIAA Committee on Standards for CFD is currently updating this Guide to incorporate in it the important developments that have taken place in $\mathrm{V} \& \mathrm{~V}$ concepts, methods, and practices, particularly with regard to the broader context of predictive capability and uncertainty quantification (UQ) methods and approaches.

This paper will provide an overview of the changes and extensions currently underway to update the AIAA Guide. Specifically, a framework for predictive capability will be described for incorporating a wide range of error and uncertainty sources identified during the modeling, verification, and validation processes, with the goal of estimating the total prediction uncertainty of the simulation. The Guide's goal is to provide a foundation for understanding and addressing major issues and concepts in predictive CFD. However, this
\end{abstract}


Guide will not recommend specific approaches in these areas as the field is rapidly evolving. It is hoped that the guidelines provided in this paper, and explained in more detail in the Guide, will aid in the research, development, and use of CFD in engineering decision-making.

\section{Introduction and Overview}

Computational fluid dynamics (CFD) and other advanced modeling and simulation (M\&S) methods are increasingly relied on for predictive performance, reliability and safety of engineering systems. Analysts, designers, decision makers, and project managers, who must depend on simulation, need practical techniques and methods for assessing simulation credibility. The AIAA Guide for Verification and Validation of Computational Fluid Dynamics Simulations (AIAA G-077-1998 (2002)), originally published in 1998, was the first engineering standards document available to the engineering community for verification and validation $(\mathrm{V} \& \mathrm{~V})$ of simulations. Much progress has been made in these areas since 1998. The AIAA Committee on Standards for CFD is currently updating this Guide to incorporate in it the important developments that have taken place in $\mathrm{V} \& \mathrm{~V}$ concepts, methods, and practices, particularly with regard to the broader context of predictive capability and uncertainty quantification (UQ) methods and approaches.

The goal of the updated AIAA Guide (Guide Update) is to provide a foundation for understanding and addressing major issues and concepts in predictive CFD. Predictive CFD is the application of CFD computations to problems or conditions for which these computations have not been validated, and is, in turn, enabled by the implementation and practice of systematic, focused and structured programs for V\&V and UQ. To that end, AIAA Guide will explain how verification, validation, and uncertainty quantification can form a comprehensive framework for estimating predictive uncertainty of M\&S. This framework is comprehensive in the sense that it treats all types of uncertainty, incorporates uncertainty due to mathematical form of the model, provides a procedure for including estimates of numerical error in the predictive uncertainty, and provides a means for propagating parameter uncertainty through the model to the system response quantity of interest. The framework, thus, provides a means to estimate and quantify the total predictive uncertainty and, in turn, a way to convey the total predictive uncertainty of a simulation result to decision makers. However, at this point the Guide will not recommend specific approaches in these areas as the field is still rapidly evolving. It is hoped that in the near term the Guide will aid in the research, development, and use of CFD as a predictive tool, especially in support of engineering decision-making.

In practice, it is envisioned that the AIAA Guide Update will educate and inform the software and methods developers, analysts, technical management and decision-makers on the value of and the need to conduct V\&V and UQ for M\&S. Specifically, the Guide Update will show:

1) Why V\&V and UQ are important and needed in support of M\&S;

2) How $V \& V$ and UQ contribute to improving the credibility of $M \& S$ and supporting decision making;

3) Who is responsible for conducting specific elements of V\&V and UQ; and

4) Guidelines for accomplishing various activities in $V \& V$ and $U Q$.

This paper proposes a framework for predictive capability that incorporates a wide range of error and uncertainty sources identified during the modeling, verification, and validation processes, with the goal of estimating the total prediction uncertainty of the M\&S. Specifically, it describes the framework and its main components and outlines the main methods and approaches for quantification of uncertainty and for propagating uncertainty in predictive M\&S. It also discusses how predictive capability relies on V\&V and UQ, as well as other factors that affect predictive capability.

The described framework will be included and featured in the upcoming AIAA Guide Update as a set of general guidelines for predictive CFD. It is envisioned that over time the AIAA Guide will foster and support the use of predictive CFD in engineering design, virtual prototyping, certification, risk analysis, and high-consequence decision-making. 


\section{Fundamental Concepts in Verification, Validation, and Uncertainty Quantification}

For scientific simulations, one starts with governing equations. These equations describe the system of interest, and encapsulate knowledge of physics, chemistry, and mathematics. The equations constitute a mathematical model of the physical world, and may be made up of submodels, or accept other submodels as input to the problem to be solved. The equations are often field equations - partial differential equations (PDEs) or integro-differential equations - that describe a time-varying solution over some volume of space or material, subject to initial and boundary conditions. Submodels could describe the particular response of a material, of interactions between materials such as chemical reactions, or some aspect of the governing equations that is approximated or makes the equations easier to solve.

The problem, then, is to find a solution to the mathematical model given the initial and boundary conditions and the appropriate submodels. One approach to obtaining solutions is through numerical simulations. The governing equations are discretized by algorithms, or numerical methods, and as a result the mathematical model is transformed to a discrete model, or a computational model. Solutions of the computational model (numerical solutions) are approximate solutions of the mathematical model. To obtain numerical solutions, the computational model is implemented in software on a computer; this implementation is often called a computer code, and an execution of the code is called a simulation. The numerical methods considered here have one or more parameters that specify the number of discrete degrees of freedom, such as the number of elements, the number of particles, or the number of discrete wave numbers, and these parameters control the accuracy and the computational cost of the simulation.

Verification and validation are the primary processes for assessing and quantifying the accuracy and reliability of numerical simulations. The V\&V definitions used in this paper are those adopted from the original AIAA Guide for Verification and Validation of Computational Fluid Dynamics Simulations (AIAA Guide) [1]:

- Verification is the process of determining that a model implementation accurately represents the developer's conceptual description of the model and the solution to the model.

- Validation is the process of determining the degree to which a model is an accurate representation of the real world from the perspective of the intended uses of the model.

In essence, verification provides evidence that the model is solved right. Verification does not address whether the model has any relationship to the real world. Verification activities only evaluate whether the model, the mathematical and computer code representation of the physical system, is solved accurately. Verification is composed of two types of activities: code verification and solution verification. Code verification deals with assessing: (a) the adequacy of the numerical algorithms to provide accurate numerical solution to the PDEs assumed in the mathematical model; and (b) the fidelity of the computer programming to implement the numerical algorithms to solve the discrete equations. Solution verification deals with the quantitative estimation of numerical accuracy of solutions to the PDEs computed by the code. The primary emphasis in solution verification is significantly different from that in code verification because there is no known exact solution to the PDEs of interest. As a result, we believe solution verification is more correctly referred to as numerical error estimation; that is, the primary goal is estimating the numerical accuracy of given solution, typically for nonlinear PDEs with singularities, discontinuities, and complex geometries. For this type of PDE, numerical error estimation is fundamentally empirical (a posteriori), i.e., the conclusions are based on evaluation and analysis of solution results from the code.

Validation, on the other hand, provides evidence that the right model is solved. This perspective implies that the model is solved correctly, or verified. However, multiple errors or inaccuracies can cancel one another and give the appearance of a validated solution. Verification is the first step of the validation process and, while not simple, it is much less complex than the more comprehensive nature of validation. Validation addresses the question of the fidelity of the model to specific conditions of the real world. The terms "evidence" and "fidelity" both imply the concept of "estimation of tolerance," not simply "yes" or "no" answers.

The goal of validation is to determine the predictive capability of a computational model for its intended use. This is accomplished by comparing computational predictions (simulation outcomes) to observations (experimental outcomes). The approach of validation is to measure the agreement between the simulation outcomes from a 
computational model and the experimental outcomes from appropriately designed and conducted experiments, i.e., validation experiment. A validation metric is the basis for comparing simulation outcomes with experimental outcomes and, as such, serves as a measure that defines the level of accuracy and precision of a simulation. Validation metrics should be established during the validation (or accuracy) requirement phase of the V\&V program planning and development. In selecting the validation metric, the primary consideration should be what the model must predict in conjunction with what types of data could be available from the experiment. Additionally, the metrics should provide a measure of agreement that includes uncertainty requirements, e.g., experimental and modeling uncertainties in dimensions, materials, loads, and responses. In most cases, assessing the predictive capability of a computational model over the full range of its intended use cannot be based solely upon data already available at the beginning of the $\mathrm{V} \& \mathrm{~V}$ program. The challenge is to define and conduct a set of experiments that will provide a stringent-enough test of the model that the decision maker will have adequate confidence to employ the model for predicting the reality of interest. If the model predicts the experimental outcomes within the predetermined accuracy requirements, the model is considered validated for its intended use.

Uncertainty Quantification is the process of characterizing all uncertainties in the model and experiment, and quantifying their effect on the simulation and experimental outcomes. UQ is a closely related activity to V\&V and essential for verifying and validating simulation models. The goal of $\mathrm{UQ}$, along with $\mathrm{V} \& \mathrm{~V}$, is to enable modelers and analysts to make precise statements about the degree of confidence they have in their simulation-based predictions. In this context, UQ can be defined as the

- Identification (Where are the uncertainties?),

- Characterization (What form they are in?),

- Propagation (How they evolve during the simulation?), and

- Analysis (What are their impacts?),

of all uncertainties in simulation models. As such, in UQ of simulation models, all significant sources of error and uncertainty in model simulations must be identified and treated to quantify their effects on the simulation results. These sources of error and uncertainty include: systematic and stochastic measurement error; ignorance; limitations of theoretical models; limitations of numerical representations of those models; limitations on the accuracy and reliability of computations, approximations, and algorithms; and human error. Specifically, UQ assesses the confidence in model predictions and allows resource allocation for fidelity improvements.

Figure 1 shows a framework from the ASME Guide for Verification and Validation in Computational Solid Mechanics (ASME Guide) [2] for organizing these different model assessment techniques, i.e., verification, validation and uncertainty quantification, with the goal of assessing accuracy of numerical simulation results for an intended use. Figure 1 also identifies the various activities and products involved in V\&V and UQ assessments along the respective modeling and experimental branches. The framework recognizes that there are numerical errors and uncertainties as well as errors and uncertainties in experimental data.

In the assessment activity of code verification, the modeler uses the computational model on a set of problems with known solutions. These problems typically have much simpler geometry, loads, and boundary conditions than the validation problems, to identify and eliminate algorithmic and programming errors. Then, in the subsequent activity of solution (calculation) verification, the version of the computational model to be used for validation problems (i.e., with the geometries, loads and boundary conditions typical of those problems) is used for identifying sufficient mesh resolution to produce an adequate solution tolerance, including the effects of finite precision arithmetic. Solution verification yields a quantitative estimate of the numerical precision and discretization accuracy for calculations made with the computational model for use in validation assessments. Thus, code and solution verification serve as the basis for estimating numerical errors and uncertainty.

Validation attempts to quantify the accuracy of the mathematical model through comparisons with experimental measurements. To that end, validation experiments are performed to generate data for assessing the accuracy of the mathematical model via simulation outcomes produced by the verified computational model. A validation experiment is a physical realization of a properly posed applied mathematics problem with initial conditions, boundary conditions, material properties, and external forces. To qualify as a validation experiment, the geometry of the object being tested (e.g., a component, subassembly, assembly, or full system), the initial conditions and the boundary conditions of the experiment, and all of the other model input parameters must be prescribed as 
completely and accurately as possible. Ideally, this thoroughness on the part of the experimenter will provide as many constraints as possible, requiring few assumptions on the part of the modeler. All of the applied loads, multiple response features, and changes in the boundary conditions should be measured; and uncertainties in the measurements should be reported.

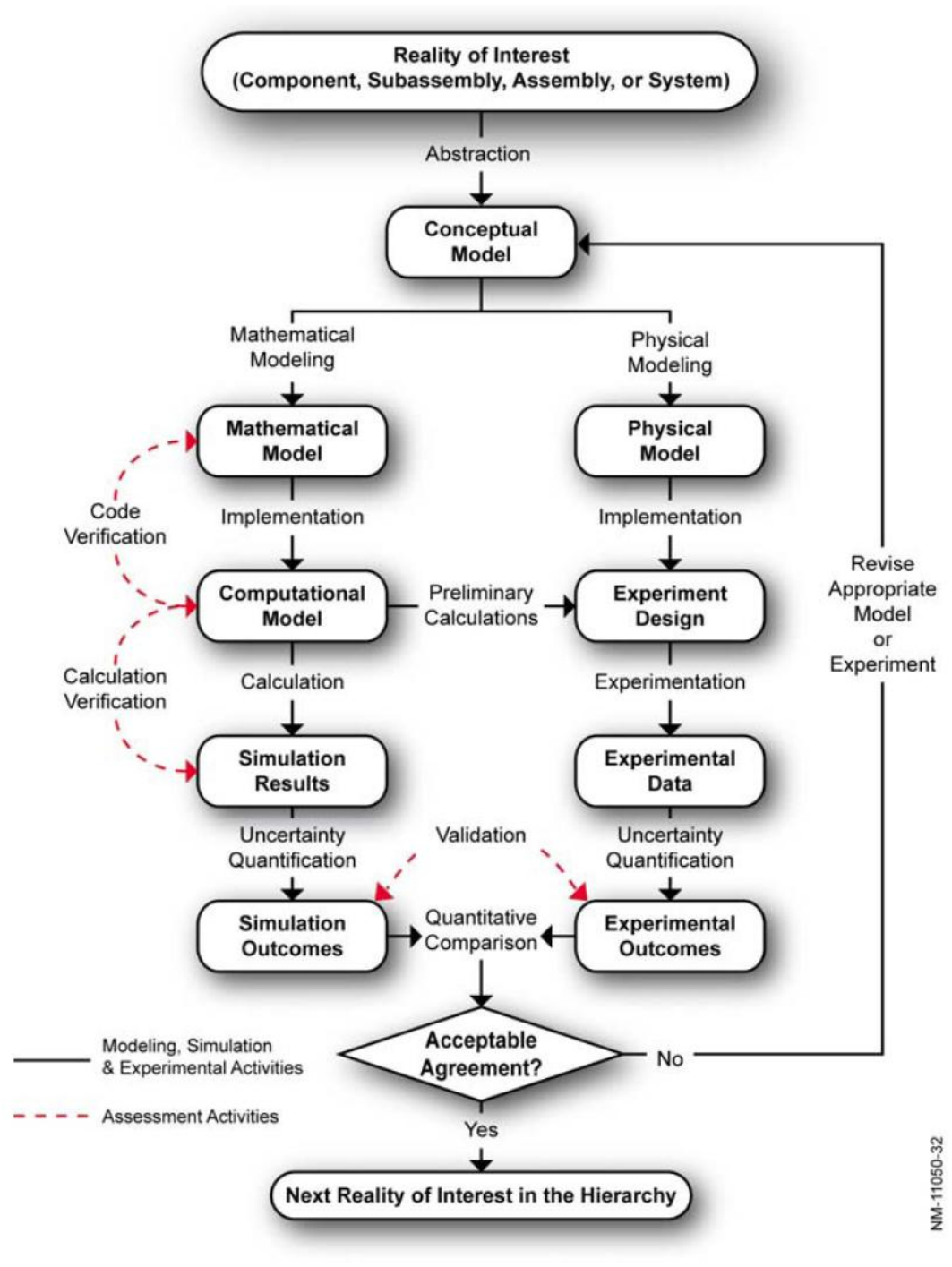

Figure 1. A framework for $V \& V$ and $U Q$ (from [2]).

Validation of mathematical models must take into account the uncertainties associated with both simulation results and experimental data. As such, throughout the modeling (left branch of Figure 1) and experimental (right branch of Figure 1) processes, and especially during the uncertainty quantification activity, all significant sources of uncertainty in model simulations and experimental measurements must be identified and treated to quantify their effects on the assessment of model accuracy, i.e., a comparison of simulation outcomes (simulation results with estimates of uncertainty) with experimental outcomes (experimental measurements with estimate of uncertainty). Model validation, thus, is the basis for quantifying model-form errors and uncertainty. Clearly, any large numerical errors or uncertainties, or large experimental errors or uncertainties, will make it difficult to isolate differences between the mathematical model and the reality it attempts to describe. As shown in Figure 1, the various assessment techniques are combined so that ultimately, a probabilistic comparison can be made between the mathematical model that the code seeks to express and the physical model that the experiments attempt to observe. 
If the agreement between the experimental and simulation outcomes is unacceptable, the model and/or the experiment can be revised. Model revision is the process of changing the basic assumptions, structure, parameter estimates, boundary values, or initial conditions of a model to improve agreement with experimental outcomes. Experiment revision is the process of changing experimental test design, procedures, or measurements to improve agreement with simulation outcomes. Whether the model or the experiment (or both) are revised depends upon the judgment of the model developer and experimenter.

In simulations involving complex physics or multidisciplinary engineering systems, strict adherence to V\&V and UQ procedures commonly becomes impractical. For example, when all of the important physical modeling parameters are not known a priori, some of the parameters are considered adjustable. Or when grid-resolved solutions are not attainable because of the computer resources needed for the simulation, adjustments must be made to improve agreement with the experimental data. When these types of activities occur, the term calibration, parameter estimation, or model updating more appropriately describes the process than does validation.

Model calibration is the process of adjusting input parameters of the model to obtain the best match between simulation results and experimental measurements. In a similar vein, model parameters may be adjusted to match results from a more sophisticated model, e.g., adjusting turbulence model parameters to match results from direct numerical simulations (DNS). Depending on the model parameter being calibrated, calibration often restricts the applicability of the model to the domain of the data used to calibrate the parameter. As such, calibration is a model development activity, but is often confused or combined with model assessment activities. For example, after calibrating a model parameter to match experimental data, a claim that the code is validated for similar conditions is often (erroneously) made. Another common practice is to refine or coarsen the mesh for a numerical simulation until the results agree with experimental data; this is a calibration of a numerical simulation parameter (the mesh resolution) but is often claimed to be validation or solution verification.

Although not shown explicitly on the modeling branch of the framework shown in Figure 1, UQ also examines the mapping between the uncertain model inputs to a code and the code's results, namely UQ estimates the effects of the input uncertainty on the simulation results. This process is known as propagation of input parameter uncertainties through the model to the output parameters to quantify the parameter uncertainty. In the context of the assessment of numerical simulation results, inputs can include parameters that define the geometry, the material models, physical models, and the boundary and initial conditions. For these types of inputs, uncertainty quantification estimates how much the simulation results may vary when these inputs are not known, are known imprecisely, or have inherent variability. Another type of input includes parameters associated with the numerical method, or possibly a choice between several methods.

\section{Perspective of Validation, Calibration, and Prediction}

The goal of model V\&V and UQ is to enable scientists and analysts to make precise statements about the degree of confidence they have in their simulation-based predictions. The AIAA Guide defines prediction as "use of a computational model to foretell the state of a physical system under conditions for which the computational model has not been validated." The predictive accuracy of the model must then reflect the strength of the inference being made from the validation database to the prediction. Thus, validation database represents reproducible evidence that a model has achieved a given level of accuracy in the solution of specified problems. From this perspective, validation comparisons do not directly allow one to make claims about the accuracy of predictions; they only allow inferences to be made. For physical processes that are well understood both physically and mathematically, the inference can be quite strong. For complex physical processes, the inference can be quite weak. The value and consequence of such an inference degrade as the physical process becomes more complex. The quantification of the inference in prediction is presently an important topic of research [3]. If necessary, one can improve the predictive accuracy of the model through additional experiments, information, and/or experience.

The requirement that the $\mathrm{V} \& \mathrm{~V}$ process quantify the predictive accuracy of the model underscores the key role of uncertainty quantification in the model-validation process. In this context, nondeterminism refers to the existence of errors and uncertainties in the outputs of computational simulations due to inherent and/or subjective uncertainties in the model. Likewise, the measurements that are made to validate these simulation outcomes also contain errors and uncertainties. While the experimental outcome is used as the reference for comparison, the V\&V process does not 
presume the experiment to be more accurate than the simulation. Instead, the goal is to quantify the uncertainties in both experimental and simulation results such that the model fidelity requirements can be assessed (validation) and the predictive accuracy of the model quantified.

Although there is increasing consistency in the formal definition of the term "validation" [1, 2], in practice there are three key, and distinct, aspects in validation [4]: (1) quantification of the accuracy of the computational model by comparing its responses with experimentally measured responses, (2) interpolation or extrapolation of the computational model to conditions corresponding to the intended use of the model, and (3) determination if the estimated accuracy of the computational model, for the conditions of the intended use, satisfies the accuracy requirements specified. The definition of validation, given at the beginning of Section 2, is not particularly clear, however, about the identification of these aspects. Consequently, this definition of validation can be interpreted to include all three aspects, or interpreted to only include the first aspect. Figure 2 depicts these three aspects, as well as the input information required by these aspects.

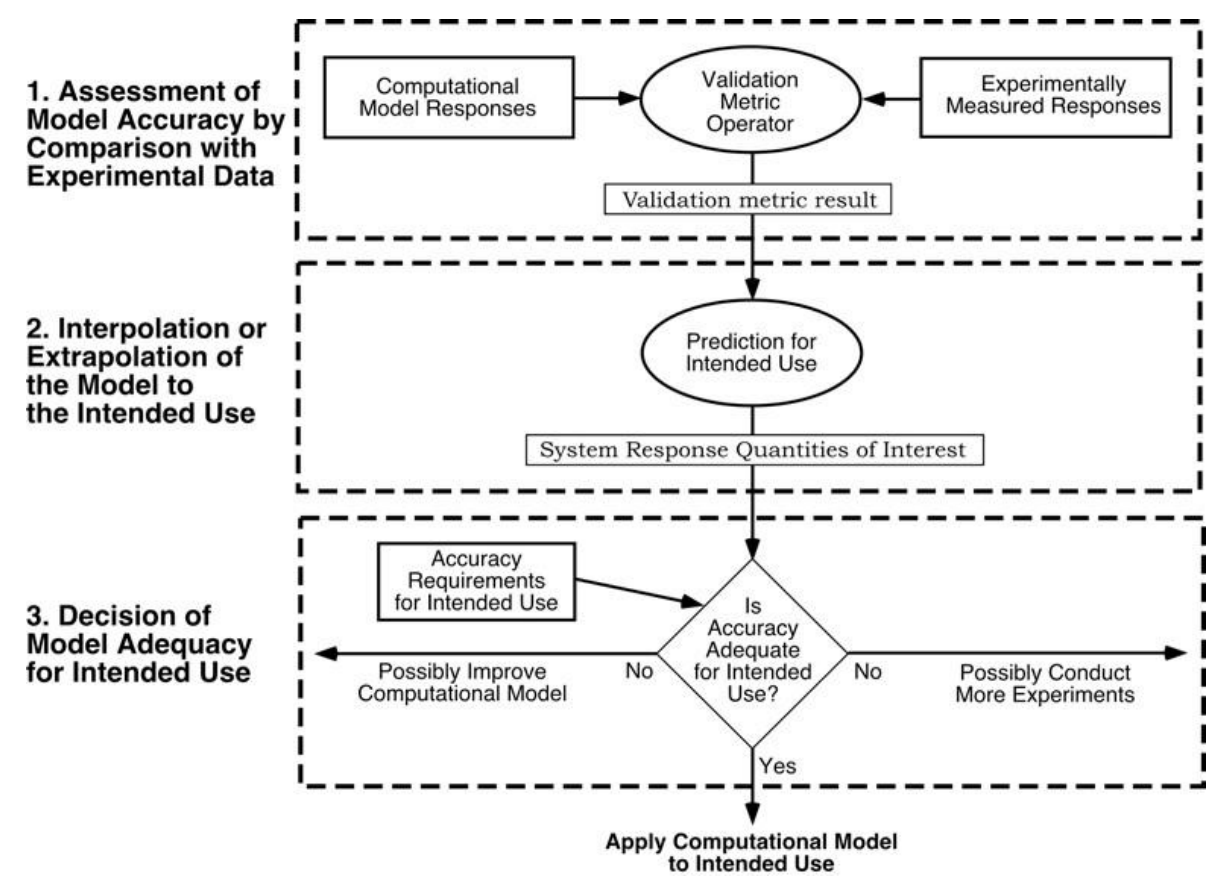

Figure 2. Three aspects of model validation (from [4]).

It is clear from Figure 2 that the quantification of model accuracy (aspect 1) obtained by comparing responses from the computational model with experimentally measured responses is distinctively different from prediction, e.g., extrapolation of the model beyond the domain of validation to the conditions of the intended use (aspect 2). The interpolation or extrapolation of the model for its intended use must include the estimated uncertainty in the prediction, which is then compared with the accuracy requirements so that a decision can be made whether the prediction accuracy is adequate (aspect 3). The most recent engineering standards document devoted to V\&V, referred to as the ASME Guide [2], considers all three aspects of validation to be fundamentally combined in the term "validation." The AIAA Guide, on the other hand, takes the view that "validation" is only concerned with the first aspect, i.e., assessment of model accuracy by comparison with experimental responses. Uncertainty is involved in this assessment, both in terms of experimental measurement uncertainty and in terms of the computational simulation, primarily because input quantities needed from the experiment either are not available or are imprecisely characterized. The second and third aspects (aspects 2 and 3) are treated in the AIAA Guide as separate activities related to predictive capability. The AIAA Guide recognizes that predictive capability uses the assessed model accuracy as input and that predictive capability also incorporates (a) additional uncertainty estimation resulting from interpolation or extrapolation of the model beyond the existing experimental database to future applications of 
interest and (b) comparison of the accuracy requirements needed by a particular application relative to the estimated accuracy of the model for that specific extrapolation to the applications of interest.

In practice, model validation can be viewed as two steps: (1) quantitatively comparing the computational and experimental results for the System Response Quantity (SRQ) of interest, and (2) determining whether there is acceptable agreement between the model and the experiment for the intended use of the model. The first step in validation deals with accuracy assessment of the model. Figure 3 depicts several important aspects of validation, as well as issues of prediction and calibration of models. The left-center portion of Figure 3 shows the first step in validation. The figure illustrates that the same SRQ must be obtained from both the computational model and the physical experiment. The SRQ can be any type of physically measurable quantity, or it can be a quantity that is based on, or inferred from, measurements. For example, the SRQ can involve derivatives, integrals, or more complex data processing of computed or measured quantities such as the maximum or minimum of functionals over a domain. The SRQs are commonly one of three mathematical forms: (1) a deterministic quantity, i.e., a single value, such as a mean value or a maximum value over a domain; (2) a probability measure, such as a probability density function or a cumulative distribution function; or (3) a probability-box (also called a p-box). Each of these forms can be functions of a parameter or multiple parameters in the computational model, such as a temperature or a Mach number; a function of spatial coordinates, such as Cartesian coordinates (x, y, z); or a function of both space and time. If both the computational and experimental SRQs are deterministic quantities, the validation metric will also be a deterministic quantity. If either of the SRQs is a probability distribution or p-box, the result of the validation metric could be a number, probability distribution, or p-box, depending on the construction of the validation metric.

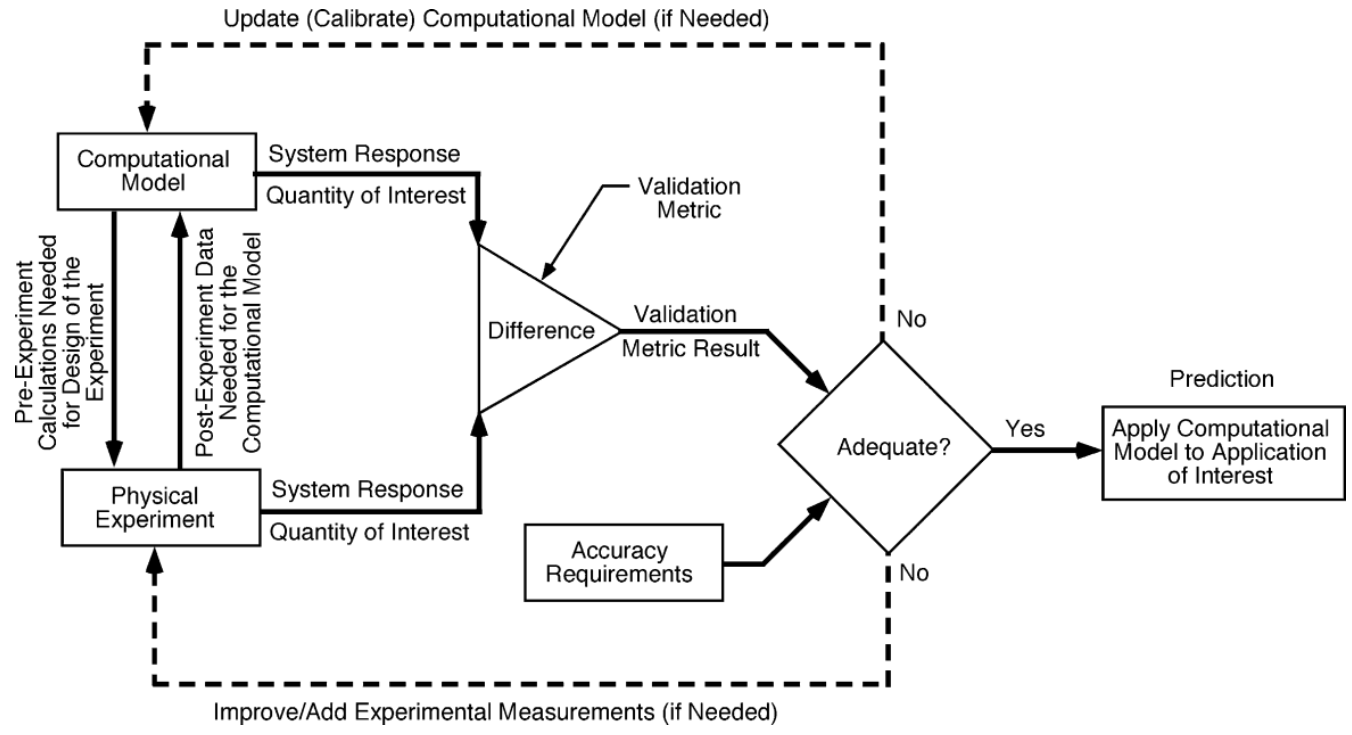

Figure 3. Validation, calibration, and prediction (from [5]).

Another feature that should be stressed in Figure 3 is the appropriate interaction between computation and experimentation that should occur in a validation experiment. To achieve the most value from the validation experiment, there should be in-depth, forthright, and frequent communication between computationalists and experimentalists during the planning and design of the experiment. Also, after the experiment has been completed, the experimentalists should measure and provide to the computationalists all the important input quantities needed to conduct the computational simulation. Examples of these quantities are actual freestream conditions attained in a wind-tunnel experiment (versus requested conditions), as-fabricated model geometry (versus as-designed), and actual deformed model geometry due aerodynamics loads and heating. What should not be provided to the computationalists in a rigorous validation activity is the measured SRQ. Stated differently, it is our view that a blind computational prediction be compared with experimental results so that a true measure of predictive capability can be assessed in the validation metric. 
The second step in validation deals with comparing the validation metric result with the accuracy requirements for the intended use of the model. That is, validation, from a practical or engineering perspective, is not a philosophical statement of truth. The second step in validation, depicted in the right-center portion of Figure 3, is an engineering decision that is dependent on the accuracy requirements for the intended use of the model. Accuracy requirements are, of course, dependent on many different kinds of factors. Some examples of these factors are: (a) the complexity of the model, the physics, and the engineering system of interest; (b) the difference in hardware and environmental conditions between the engineering system of interest and the validation experiment; (c) the increase in uncertainty due to extrapolation of the model from the validation conditions to the conditions of the intended use; (d) the risk tolerance of the decision makers involved; and (e) the consequence of failure or underperformance of the system of interest.

The right-center portion of Figure 3 shows the comparison of the validation metric result with the preliminary accuracy requirement for the application of interest. This is referred to as a preliminary requirement because, as shown in Figure 4, the conditions of application of interest, i.e., the application domain, may be different from the validation domain. If they are different, as is the usual case, then only preliminary accuracy requirement can be applied. If the model passes these preliminary accuracy requirements, then the model prediction would be made using extrapolation or interpolation of the model. Accuracy requirements for the application, i.e., final accuracy requirements, would be made after the model has been extrapolated or interpolated to the conditions of the application of interest. Setting preliminary accuracy requirements is also useful if the preliminary model accuracy is inadequate. For instance, it may be concluded that the assumptions and approximations made in the conceptual model are inadequate to achieve the accuracy needed. This may necessitate a reformulation of the model, as opposed to calibration of model parameters, at a later stage. In addition, preliminary accuracy assessment provides a natural mechanism for adjusting and adapting the final accuracy requirements to cost and schedule requirements of the engineering system of interest. For example, trade-offs can be made between required final accuracy, system design, and performance, in addition to cost and schedule.

If the preliminary accuracy requirements are not met, then one has two options, as shown in Figure 3. First, the dashed-line upper feedback loop provides for any of the activities referred to as model updating, model calibration, or model tuning. Depending on the ability to determine parameters, either by direct measurement or by inference from a model, we have divided parameter updating into three categories: parameter measurement, parameter estimation, and parameter calibration. If the model parameters are updated, one could proceed again and perform the comparison via the validation metric. It is now very likely that the validation metric result would be significantly reduced, implying much better agreement between the model and the experimental results. The parameter updating may be physically justifiable, or it may simply be expedient. How to determine the scientific soundness of updating, and its impact on predictive capability, is a very difficult issue. Second, the lower feedback loop could be taken if: (a) improvements or changes are needed in experimental measurements, such as improved diagnostic techniques; (b) additional experiments are needed to reduce experimental uncertainty; or (c) additional experiments are needed that are more representative of the application of interest.

The right portion of Figure 3 deals with the issue of use of the model to make prediction for the conditions of the application of interest. It should be noted that Figure 3 is completely consistent with the diagram showing the three aspects of validation (Figure 2). Also, Figure 3 is conceptually consistent with the ASME Guide diagram in Figure 1. To better understand the relationship between validation and prediction, consider Figure 4. The validation domain in the figure suggests three features. First, in this region we have high confidence that the relevant physics is understood and modeled at a level that is commensurate with the needs of the application. Second, this confidence has to be quantitatively demonstrated by satisfactory agreement between simulations and experiments in the validation database for some range of applicable parameters in the model. And third, the boundary of the domain indicates that outside this region there is degradation in confidence in the quantitative predictive capability of the model. Stated differently, outside the validation domain the model is credible, but its quantitative capability has not been demonstrated. The application domain, sometimes referred to as the operating envelope of the system, is generally larger than the validation domain and indicates the region where predictive capability is needed from the model for the applications of interest.

It is generally too expensive (or even impossible) to obtain experimental data over the entire multidimensional space of model input parameters for the application of interest. In many cases, it is not even possible to obtain experimental data at the application conditions of interest. As a result, the more common case is that the model 
must be applied at conditions where there are no experimental data. The process of applying the model to application conditions of interest outside of the validation domain is called model extrapolation. In practice, the inference from the validation domain to application domain regarding predictive capability of the model is made using both physics-based models and statistical methods [3]. The need to perform this extrapolation reinforces our need for models to be judged to achieve the right answer for the right reasons in the validation regime. Model calibration, which employs explicit tuning or updating of model parameters to achieve some degree of agreement with existing validation experiments, does not fully assess uncertainty in the predictive use of the model.

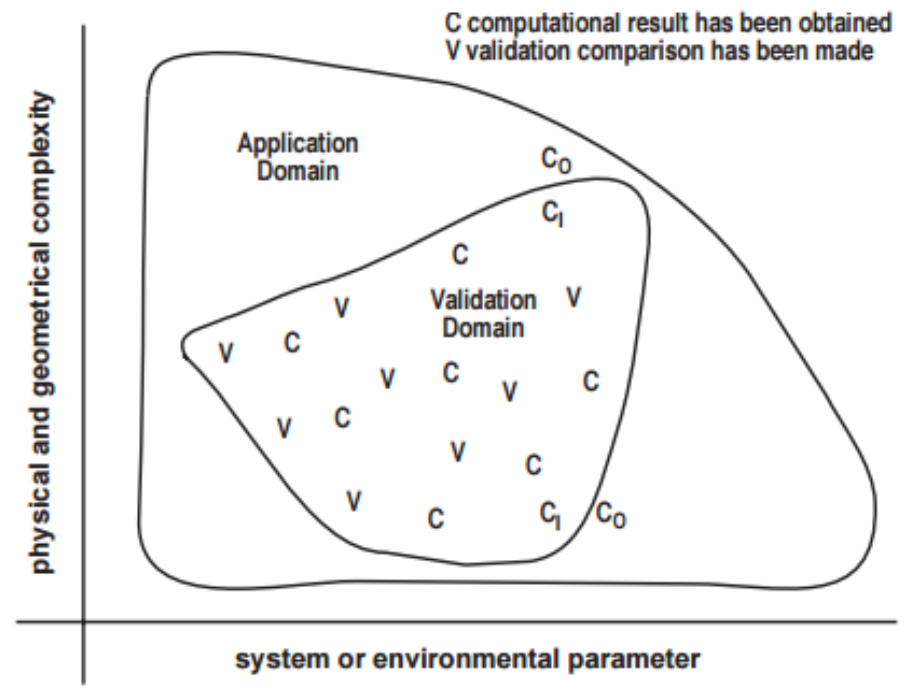

Figure 4. Typical relationship between the application domain and the validation domain (from [6]).

Given the context as explained above, if one provides a simulation-based prediction, can an estimate of the accuracy of that prediction be made? What are the sources of error and uncertainty in the prediction? That is, why might the predictive result of a simulation be different from the actual behavior of a device or system? To properly deal with the issue of predictive capability requires a detailed examination of errors and uncertainty associated with simulation-based predictions in the context of the intended application.

Verification, validation, and uncertainty quantification, as described in this paper and in the AIAA Guide Update, form a comprehensive framework for estimating predictive uncertainty of M\&S. The framework is comprehensive in the sense that it treats all types of uncertainty, incorporates uncertainty due to mathematical form of the model, provides a procedure for including estimates of numerical error in the predictive uncertainty, and provides a means for propagating parameter uncertainty through the model to the SRQ of interest. This framework, thus, provides a means to estimate and quantify the total predictive uncertainty and, in turn, a way to convey the total predictive uncertainty of a simulation result to decision makers.

\section{Predictive Capability and Uncertainty Quantification}

Uncertainty quantification, in the context of applied scientific computing, is perhaps best defined as the process of establishing the uncertainty in specific SRQ of interest in a computation from the uncertainties in a specific set of inputs to the computation, or from unknown effects or limitations in the computation. An example would be quantifying the uncertainty in the drag coefficient from the uncertainties in the operating conditions and the uncertainty due to shortcomings or limitations in the mathematical model chosen for use in the simulation of a physical system. This mode of uncertainty quantification is usually referred to as the forward mode. The inverse mode or "calibration" mode, in which the uncertainties in inputs are estimated from the uncertainties in outputs, is another form of uncertainty quantification. A more specific use of uncertainty quantification arises in the context of predictive computations, in which a scientific computing code is used outside its range of satisfactory validation (or 
outside its validation domain). This more specific meaning of uncertainty quantification is best defined as predictive uncertainty, and this more specific meaning is the one covered in this paper and targeted in the AIAA Guide Update.

It is widely understood and accepted that uncertainties, whether random or systematic, arise because of the inherent randomness in physical systems, modeling idealizations, experimental variability, measurement inaccuracy, etc., and cannot be ignored. This fact complicates the already difficult process of model validation by creating an unsure target - a situation in which neither the simulated nor the observed behavior of the system is known with certainty. In this context, nondeterminism refers to the existence of errors and uncertainties in the results of M\&S because of inherent and/or subjective uncertainties in the model inputs or model form. Likewise, the measurements that are made to validate these simulation outcomes also contain errors and uncertainties. In fact, while the experimental outcome is used as the reference, the $\mathrm{V} \& \mathrm{~V}$ process does not presume the experiment to be more accurate than the simulation. Instead, the goal is to quantify the uncertainties in both experimental and simulation results such that the model requirements can be assessed and the predictive accuracy of the model quantified.

Once code verification has established that a code correctly implements the numerical methods as intended, there remain three sources of error and uncertainty in predictions from M\&S. The first is numerical methods as they provide approximate solutions to the governing equations (numerical uncertainty). The second source of error or uncertainty is that the inputs needed for the simulation are not known precisely (parameter uncertainty). For example, the device to be simulated can only be constructed to within manufacturing tolerances; parameters in a model or submodel may not be known precisely, or may be subjective estimates from experts. A third source of error and uncertainty is that the governing equations do not adequately describe the physics; the real device or system is affected by some phenomena that are not included in the model or submodels (model form uncertainty). Different model assessment techniques - solution verification, validation, and uncertainty quantification - are used to estimate the errors and uncertainty from these three different sources. In any simulation, all three sources of error and uncertainty are present and contribute to the uncertainty in the prediction. From this it should be clear that estimating errors or uncertainty from just a single source, e.g., performing uncertainty quantification without validation or any form of verification, cannot provide the total uncertainty in a prediction.

Eliminating uncertainty in a prediction is neither practical nor necessary. It is sufficient to control, or manage, the uncertainty to an acceptable level for making the decisions that motivated the simulations. If the level of uncertainty is too high, the different assessments identify the largest sources of uncertainty and indicate where effort should be spent to reduce the uncertainty, e.g., reducing discretization error, improving physics modeling, or more precisely defining the problem. In addition, an understanding of the sources of the uncertainty can provide guidance on how to reduce uncertainty in the prediction in the most efficient and cost effective manner. Information on the magnitude, composition, and sources of uncertainty in simulation predictions is critical in the decision-making process for natural and engineered systems. Without forthrightly estimating and clearly presenting the total uncertainty in a prediction, decision makers are ill advised, possibly resulting in inadequate safety, reliability, and performance of the system.

\subsection{Types of Uncertainty}

Uncertainty and error can be categorized as error, irreducible uncertainty, and reducible uncertainty. Errors create a reproducible (i.e., deterministic) bias in the prediction and can theoretically be reduced or eliminated. Errors can be acknowledged (detected) or unacknowledged (undetected). Examples include inaccurate model form, implementation errors in the computational code, nonconverged computational models, etc. While there are many different ways to classify uncertainty, we will use the taxonomy prevalent in the risk assessment community that categorizes uncertainties according to their fundamental essence [7 - 10]. Thus, uncertainty is classified as either a) aleatory - the inherent variation in a quantity that, given sufficient samples of the stochastic process, can be characterized via a probability distribution, or b) epistemic - where there is insufficient information concerning the quantity of interest to specify either a fixed value or a precisely known probability distribution. In scientific computing, there are many sources of uncertainty including the model inputs, the form of the model, and poorlycharacterized numerical approximation errors. All of these sources of uncertainty can be classified as either purely aleatory, purely epistemic, or a mixture of aleatory and epistemic uncertainty. 


\subsubsection{Aleatory Uncertainty}

Aleatory uncertainty (also called irreducible uncertainty, stochastic uncertainty, or variability) is uncertainty due to inherent variation or randomness and can occur among members of a population or due to spatial or temporal variations. Aleatory uncertainty is generally characterized by a probability distribution, most commonly as either a probability density function (PDF) - which quantifies the probability density at any value over the range of the random variable - or a cumulative distribution function (CDF) - which quantifies the probability that a random variable will be less than or equal to a certain value (see Figure 5). Here we will find it more convenient to describe aleatory uncertainties with CDFs. An example of an aleatory uncertainty is a manufacturing process that produces parts that are nominally 0.5 meters long. Measurement of these parts will reveal that the actual length for any given part will be different than 0.5 meters. With sufficiently large number of samples, both the form of the CDF and the parameters describing the distribution of the population can be determined. The aleatory uncertainty in the manufactured part can only be changed by modifying the fabrication or quality control processes; however, for a given set of processes, the uncertainty due to manufacturing is considered irreducible.

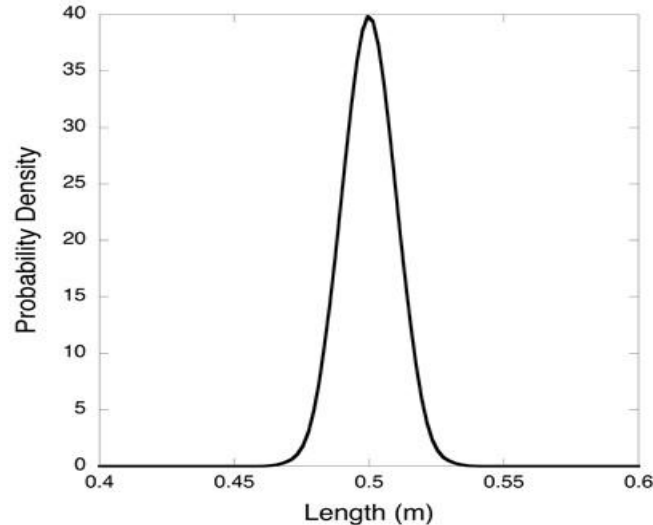

a) probability density function (PDF)

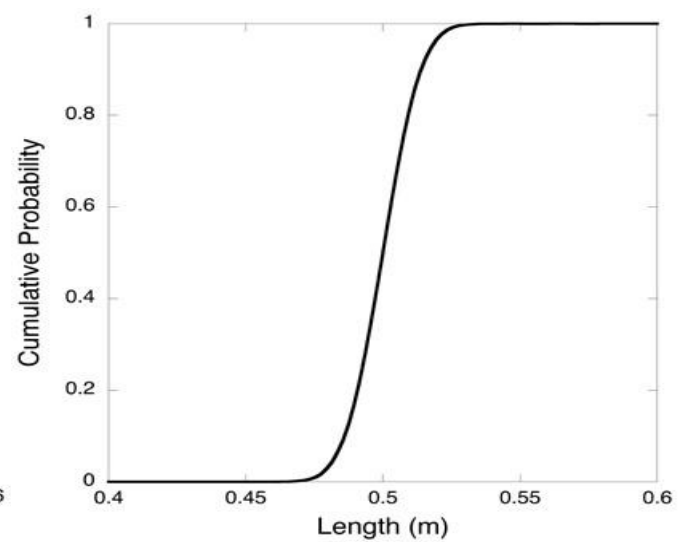

b) cumulative distribution function $(\mathrm{CDF})$

Figure 5. Example of probability distributions.

\subsubsection{Epistemic Uncertainty}

Epistemic uncertainty (also called reducible uncertainty or ignorance uncertainty) is uncertainty that arises due to a lack of knowledge on the part of the analyst, or team of analysts, conducting the modeling and simulation. If knowledge is added (through experiments, improved numerical approximations, expert opinion, higher fidelity physics modeling, etc.) then the uncertainty can be reduced. If sufficient knowledge is added, then the epistemic uncertainty can, in principle, be eliminated. Epistemic uncertainty is traditionally represented as either an interval with no associated probability distribution or a probability distribution that represents degree of belief of the analyst, as opposed to frequency of occurrence discussed in aleatory uncertainty. In this paper, we will represent epistemic uncertainty as an interval-valued quantity, meaning that the true (but unknown) value can be any value over the range of the interval, with no likelihood or belief that any value is more true than any other value. The Bayesian approach to uncertainty quantification characterizes epistemic uncertainty as a probability distribution that represents the degree of belief on the part of the analyst [11-13].

The distinction between aleatory and epistemic uncertainty is not always easily determined during characterization of input quantities or the analysis of a system. For example, consider the manufacturing process mentioned above, where the length of the part is described by a probability distribution, i.e., it is an aleatory uncertainty. However, if we are only able to measure a small number of samples (e.g., three) from the population, then we will not be able to accurately characterize the probability distribution. In this case, the uncertainty in the length of the parts could be characterized as a combination of aleatory and epistemic uncertainty. By adding information, i.e., by measuring more samples of manufactured parts, then the probability distribution (both its form and its parameters) could be more accurately characterized. When one obtains a large number of samples, then one can characterize the uncertainty in length as a purely aleatory uncertainty given by a precise probability distribution, i.e., a probability distribution with fixed values for all of the parameters than define the chosen distribution. 
In addition, the classification of uncertainties as either aleatory or epistemic depends on the question being asked. In the manufacturing example given above, if one asks "What is the length of a specific part produced by the manufacturing process?" then the correct answer is a single true value that is not known, unless the specific part is accurately measured. If instead, one asks "What is the length of any part produced by the manufacturing process?" then the correct answer is that the length is a random variable that is given by the probability distribution determined using the measurement information from a large number of sampled parts.

\subsection{Sources of Uncertainty}

For a complete uncertainty quantification framework, all of the possible sources of uncertainty must be identified and characterized. When fixed values are known precisely (or with very small uncertainty), then they can be treated as deterministic. Otherwise, they should be classified as either aleatory or epistemic and characterized with the appropriate mathematical representation. Sources of uncertainty can be broadly categorized as occurring in model inputs, numerical approximations, or in the form of the mathematical model. We will briefly discuss each of these categories below; see Ref. [3] for a complete description.

\subsubsection{Model Inputs}

Model inputs include not only parameters used in the model of the system, but also data from the surroundings (see Figure 6). Model input data includes things such as geometry, constitutive model parameters, and initial conditions, and can come from a range of sources including experimental measurement, theory, other supporting simulations, or even expert opinion. Data from the surrounding includes boundary conditions and system excitation (mechanical forces or moments acting on the system, forcing fields such as gravity and electromagnetism, etc.). Uncertainty in model inputs can be either aleatory or epistemic.

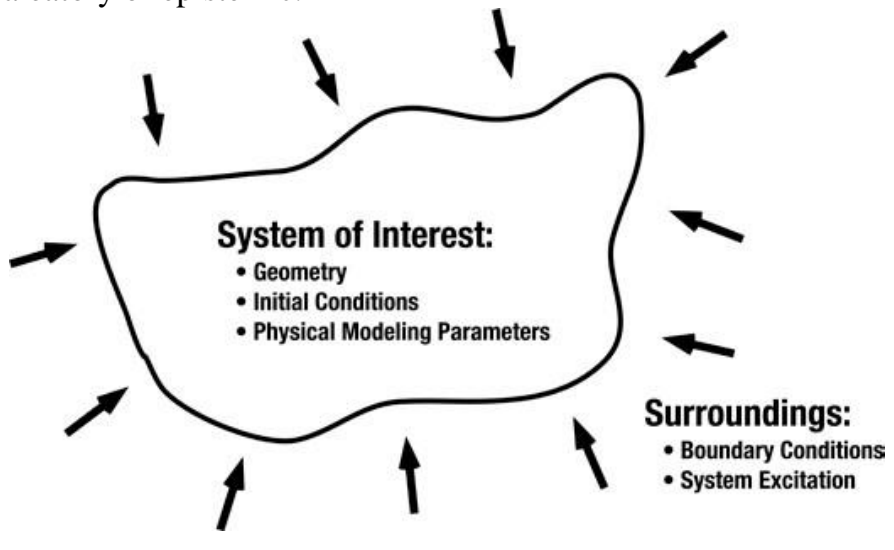

Figure 6. Schematic of model input uncertainty (from [3]).

\subsubsection{Numerical Approximation}

Since complicated differential equation-based models rarely admit exact solutions for practical problems, approximate numerical solutions must be used. The characterization of the numerical approximation errors associated with a simulation is called verification [1, 2]. It includes discretization error, iterative convergence error, round-off error, and also errors due to coding mistakes. Discretization error arises due to the fact that the spatial domain is decomposed into a finite number of nodes/elements and, for unsteady problems, time is advanced with a finite time step. Discretization error is one of the most difficult numerical approximation errors to estimate and is also often the largest of the numerical errors. Iterative convergence errors are present when the discretization of the model results in a simultaneous set of algebraic equations that are solved approximately or when relaxation techniques are used to obtain a steady-state solution. Round-off errors occur due to the fact that only a finite number of significant figures can be used to store floating point numbers on digital computers. Finally, coding mistakes can occur when numerical algorithms are implemented into a software tool. Since coding mistakes are, by definition, unknown errors (they are generally eliminated when they are identified), their effects on the numerical solution are extremely difficult to estimate. 
For cases where numerical approximation errors can be estimated, their impact on the SRQs of interest can be eliminated, given that sufficient computing resources are available. If this is not possible, they should generally be converted to epistemic uncertainties due to the uncertainties associated with the error estimation process itself. Some researchers argue that numerical approximation errors can be treated as random variables and that the variance of the contributors can be summed to obtain an estimate of the total uncertainty due to numerical approximations [14, 15]. We believe this approach is unfounded and that traditional statistical methods cannot be used. Estimates of numerical approximation errors are analogous to bias (systematic) errors in experimental measurements; not random measurement errors. As is well known, bias errors are much more difficult to identify and quantify than random errors.

\subsubsection{Model Form}

The form of the model results from all assumptions, conceptualizations, abstractions, and mathematical formulations on which the model relies such as ignored physics or physics coupling in the model [3]. The characterization of model form uncertainty is commonly estimated in model validation. Since the term validation can have different meanings in various communities, we expressly define it to be: assessment of model accuracy by way of comparison of simulation results with experimental measurements. This definition is consistent with Refs. [1, 2]. Although model validation has been a standard procedure in science and engineering for over a century, our approach takes two additional steps. First, it statistically quantifies the disagreement between the simulation results and all of the conditions for which experimental measurements are available. Second, it extrapolates this error structure from the domain of available experimental data to application conditions of interest where experimental data are not available. This approach was presented in Ref. [16]. In this approach, model form uncertainty is treated as epistemic.

It should be noted that the experimental data used for model validation also contains aleatory uncertainty, and may include significant epistemic uncertainty due to unknown bias errors. While there are well-established methods for treating aleatory uncertainty in experimental data (e.g., see Ref. [17]), it is generally the bias errors that are most damaging to model validation efforts. Figure 7 presents the astronomical unit (the mean distance between the earth and the sun) as measured by various researchers over time, along with their estimated uncertainty [18]. It is striking to note that in each case, the subsequent measurement falls outside the uncertainty bounds given for the previous measurement. This example suggests that epistemic uncertainty due to bias errors are generally underestimated (or neglected) in experimental measurements. When possible, methods should be employed that convert correlated bias errors into random uncertainties by using techniques such as Design of Experiments [19-21].

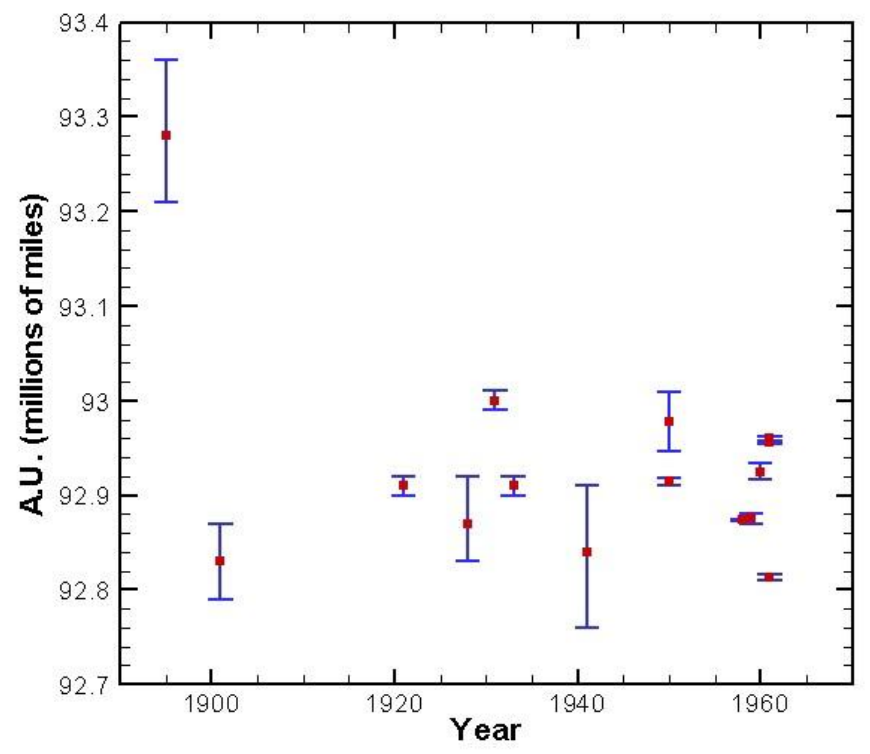

Figure 7. Measurements of the Astronomical Unit (A.U.) over time including estimated uncertainty (data from [18]). 


\subsection{Uncertainty Framework}

This section gives a broad overview of a complete framework for assessing the predictive uncertainty of scientific computing applications to be included in the upcoming AIAA Guide Update. The framework is complete in the sense that it treats both types of uncertainty (aleatory and epistemic) and incorporates uncertainty due to the form of the model and any numerical approximations used. Only a high-level description of this uncertainty framework is given here, additional details can be found in Ref. [3]. A novel aspect of the framework is that it addresses all sources of uncertainty in scientific computing including uncertainties due to the form of the model and numerical approximations. The purpose of this framework is to be able to estimate the uncertainty in a SRQ for which no experimental data are available. That is, the mathematical model, which embodies approximations to the relevant physics, is used to predict the uncertain SRQ, which includes input uncertainties, numerical approximation uncertainties, and an extrapolation of the model form uncertainty to the conditions of interest. The basic steps in the uncertainty framework are described next, with emphasis on aspects of the framework that are new in the field of predictive uncertainty.

\subsubsection{Identify All Sources of Uncertainty}

All potential sources of uncertainty in model inputs must be identified. If an input is identified as having little or no uncertainty, then it is treated as deterministic, but such assumptions must be justified and understood by the decision maker using the simulation results. The goals of the analysis should be the primary determinant for what is considered as fixed versus what is considered as uncertain. The general philosophy that should be used is: consider an aspect as uncertain unless there is a strong and convincing argument that the uncertainty in the aspect will result in minimal uncertainty in all of the SRQs of interest in the analysis.

As discussed earlier, sources of uncertainty are categorized as occurring in model inputs, numerical approximations, or in the form of the mathematical model. We point out that there are types of model form uncertainty that are difficult to identify. These commonly deal with assumptions in the conceptual model formulation or cases where there is a very large extrapolation of the model. Some examples are a) assumptions concerning the environment (normal, abnormal, or hostile) to which the system is exposed, b) assumptions concerning the particular scenarios the system is operating under, e.g., various types of damage or misuse of the system, and c) cases where experimental data are only available on subsystems, but predictions of complete system performance are required. Sometimes, separate simulations are conducted with lower-fidelity models (using different assumptions) to help identify the additional sources of uncertainty in the model inputs or the model form.

\subsubsection{Characterize Uncertainties}

By characterizing a source of uncertainty we mean a) assigning a mathematical structure to the uncertainty and b) determining the numerical values of all of the needed elements of the structure. Stated differently, characterizing the uncertainty requires that a mathematical structure be given to the uncertainty and all parameters of the structure be numerically specified such that the structure represents the state of knowledge of every uncertainty considered. The primary decision to be made concerning the mathematical structure for each source is: should it be represented as a purely aleatory uncertainty, a purely epistemic uncertainty, or a mixture of the two?

For purely aleatory uncertainties, the uncertainty is characterized as a precise probability distribution, i.e., a CDF is given with fixed quantities for each of the parameters of the chosen distribution. For purely epistemic uncertainties, such as numerical approximations and model form, the uncertainty is characterized as an interval. For an uncertainty that is characterized as a mixture of aleatory and epistemic uncertainty, then an imprecise probability distribution is given. This mathematical structure is a probability distribution with interval-valued quantities for the parameters of the distribution. This structure represents the ensemble of all probability distributions that exist whose parameters are bounded by the specified intervals. This structure commonly arises in characterization of information from expert opinion. For example, suppose a new manufacturing process is going to be used to produce a component. Before inspection samples can be taken from the new process, a manufacturing expert could characterize its features or performance as an imprecise probability distribution.

\subsubsection{Estimate Uncertainty due to Numerical Approximation}

Recall that the sources of numerical approximation error include discretization, incomplete iteration, and round off. Methods for estimating discretization error include Richardson extrapolation [24], discretization error transport 
equations [25, 26], and residual/recovery methods in finite elements [27 - 29]. Regardless of the approach used for estimating the discretization error, the reliability of the estimate depends on the solutions being in the asymptotic grid convergence range [3,30], which is extremely difficult to achieve for complex scientific computing applications. Various techniques are available for estimating iterative convergence errors (e.g., see Ref. [3]). Roundoff errors are usually small, but can be reduced if necessary by increasing the number of significant figures used in the computations (e.g., by going from single to double precision). Since errors due to the presence of unknown coding mistakes or algorithm inconsistencies are difficult to characterize, their effects should be minimized by employing good software engineering practices and using specific techniques for scientific computing software such as order of accuracy verification (e.g., see Refs. [3, 24, 31]).

Because of the difficulties of obtaining accurate estimates of the different numerical approximation errors, in most cases they should be converted to and explicitly represented as epistemic uncertainties. The simplest method for converting error estimates to uncertainties is to use the magnitude of the error estimate to apply bands above and below the fine grid simulation prediction, possibly with an additional factor of safety included. For example, Roache's Grid Convergence Index [24, 32] is a method for converting the discretization error estimate from Richardson extrapolation to an uncertainty. The resulting uncertainty is epistemic since additional information (i.e., grid levels, iterations, digits of precision) could be added to reduce it. When treating epistemic uncertainties as intervals, the proper mathematical method for combining uncertainties due to discretization $\left(U_{D E}\right)$, incomplete iteration $\left(U_{I T}\right)$, and round off $\left(U_{R O}\right)$ is to simply sum the uncertainties.

$$
U_{N U M}=U_{D E}+U_{I T}+U_{R O}
$$

It can easily be shown that $U_{N U M}$ is a guaranteed bound on the total numerical error, given that each contributor is an accurate bound.

Implementing Eq. (1) in practice is a tedious and demanding task, even for relatively simple simulations because of two features. First, Eq. (1) must be calculated for each SRQ of interest in the simulation. For example, if the SRQs are pressure, temperature, and three velocity components in a flowfield, then that $U_{N U M}$ should be calculated for each quantity over the domain of the flow field. Second, each of the SRQs varies as a function of the uncertain input quantities in the simulation. One common technique for limiting the computational effort involved in making all of these estimates is to determine the locations in the domain of the partial differential equations and the conditions for which the input uncertainties are believed to produce the largest values of $U_{D E}, U_{I T}$, and $U_{R O}$. The resulting uncertainties are then applied over the entire physical domain and application space. This simplification is a reasonable approach, but it is not always reliable because of potentially large variations in the SRQs over the domain of the differential equation, and because of nonlinear interactions between input uncertainties.

\subsubsection{Propagate Input Uncertainties through the Model (Estimation of Parameter Uncertainty)}

When the uncertainties in the model input parameters have been established, these uncertainties can be propagated through the model to establish expected uncertainties on the simulation output quantities. Methods for calculating and propagating input uncertainties in simulation codes can be divided into probabilistic methods and nonprobabilistic methods. The former employ or assume that variables (whether inputs or outputs) have a known or discernible PDF, while the latter do not assume any knowledge of the PDF. Probabilistic methods for propagating uncertainties can be further divided into sampling methods and methods that use spectral expansions of the probabilistic variations.

When probabilistic or nondeterministic methods are used to propagate input uncertainties through the model, then the numerical convergence of the propagation technique itself must also be considered. The key issue in nondeterministic simulations is that a single solution to the mathematical model is no longer sufficient. A set, or ensemble, of calculations must be performed to map the uncertain input space to the uncertain output space. Sometimes, this is referred to as ensemble simulations instead of nondeterministic simulations. Figure 8 depicts the propagation of input uncertainties through the model to obtain output uncertainties. The number of individual calculations needed to accurately accomplish the mapping depends on four key factors: a) the nonlinearity of the partial differential equations, b) the dependency structure between the uncertain quantities, c) the nature of the uncertainties, i.e., whether they are aleatory or epistemic uncertainties, and d) the numerical methods used to compute the mapping. The number of mapping evaluations, i.e., individual numerical solutions of the mathematical 
model, can range from tens to hundreds of thousands. As noted, many techniques exist for propagating input uncertainties through the mathematical model to obtain uncertainties in the SRQs.

There are commonly one or more system outputs (i.e., SRQs) that the analyst is interested in predicting with a simulation code. When uncertain model inputs are aleatory, there are a number of different approaches for propagating this uncertainty through the model. The simplest approach is sampling (e.g., Monte Carlo) where inputs are sampled from their probability distribution and then used to generate a sequence of SRQs; however, sampling methods tend to converge slowly as a function of the number of samples. Other approaches that can be used to propagate aleatory uncertainty include perturbation methods and polynomial chaos (both intrusive and non-intrusive formulations). Furthermore, when a response surface approximation of an SRQ as a function of the uncertain model inputs is available, then any non-intrusive method discussed above (including sampling) can be computed efficiently.

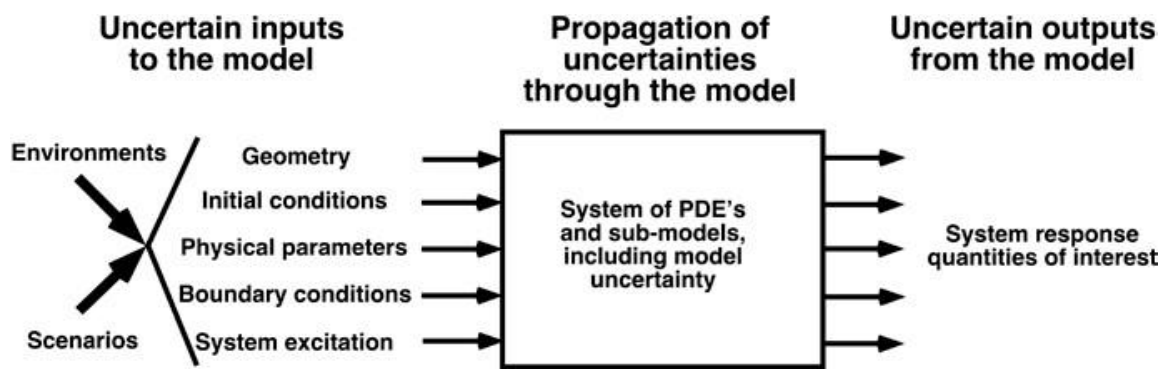

Figure 8. Propagation of input uncertainties to obtain output uncertainties (from [3]).

When all uncertain inputs are characterized by intervals, i.e., they are purely epistemic, there are two popular approaches for propagating these uncertainties to the SRQs. The simplest is sampling over the input intervals to estimate the interval bounds of the SRQs. However, the propagation of interval uncertainty can also be formulated as a bound-constrained optimization problem: given the possible interval range of the inputs, determine the resulting minimum and maximum values of the SRQs. Thus, standard approaches for constrained optimization such as local gradient-based searches and global search techniques can be used.

When some uncertain model inputs are aleatory and others are epistemic, then a segregated approach to uncertainty propagation should be used [22, 41]. For example, in an outer-loop, samples from the epistemic uncertain model inputs may be drawn. For each of these sample-values, the aleatory-uncertain model inputs are propagated assuming a fixed sample-value of the epistemically-uncertain quantity. The completion of each step in the outer loop results in a possible (because of limited knowledge) CDF of the SRQ. The end result of the segregated uncertainty propagation process will be an ensemble of possible CDFs of the SRQ, the outer bounding values of which are used to form a p-box. Note that the bounding values of the p-box come from any combination of individual CDFs, or portions of individual CDFs, that were generated. An advantage of this segregated approach is that the inner aleatory uncertainty propagation loop can be achieved using any of the techniques described above for propagating probabilistic uncertainty (i.e., it is not limited to simple sampling approaches).

An example of Monte Carlo sampling method used for propagating both aleatory and epistemic uncertainty in model inputs through the model to determine the effects on the SRQ is described below. As noted, although both aleatory and epistemic uncertainties can be propagated using sampling methods, they must each be treated independently because they characterize two different types of uncertainty. Sampling an aleatory uncertainty implies a sample is taken from a random variable and that each sample is associated with a probability. Sampling an epistemic uncertainty implies a sample is taken from a range of possible values. The sample has no probability or frequency of occurrence associated with it; we only know it is possible, given the information available concerning the input quantity.

\subsubsection{Aleatory Uncertainty}

Recall that aleatory uncertainties are represented with a CDF (e.g., see Figure 5b). For Monte Carlo sampling, a sample is chosen between 0 and 1 based on a uniform probability distribution. Then this probability is mapped using the CDF characterizing the input uncertainty to determine the corresponding value of the input quantity (see top of 
Figure 9). When more than one uncertain input is present (e.g., $x_{1}, x_{2}$, and $x_{3}$ ), Monte Carlo sampling randomly (and independently) picks probabilities for each of the input parameters as shown in Figure 9. Once the input parameter samples are chosen, the model is used to compute a SRQ $(y)$ for each sample. This sequence of SRQs is then ordered from smallest to largest, making up the abscissa of the CDF of the SRQ. The ordinate is found by separating the corresponding probabilities into equally-spaced divisions, where each division has a probability of $1 / N$, where $N$ is the total number of Monte Carlo samples (see bottom of Figure 9). The CDF of the SRQ is the mapping of the uncertain inputs through the model to obtain the uncertainty in the model outputs.

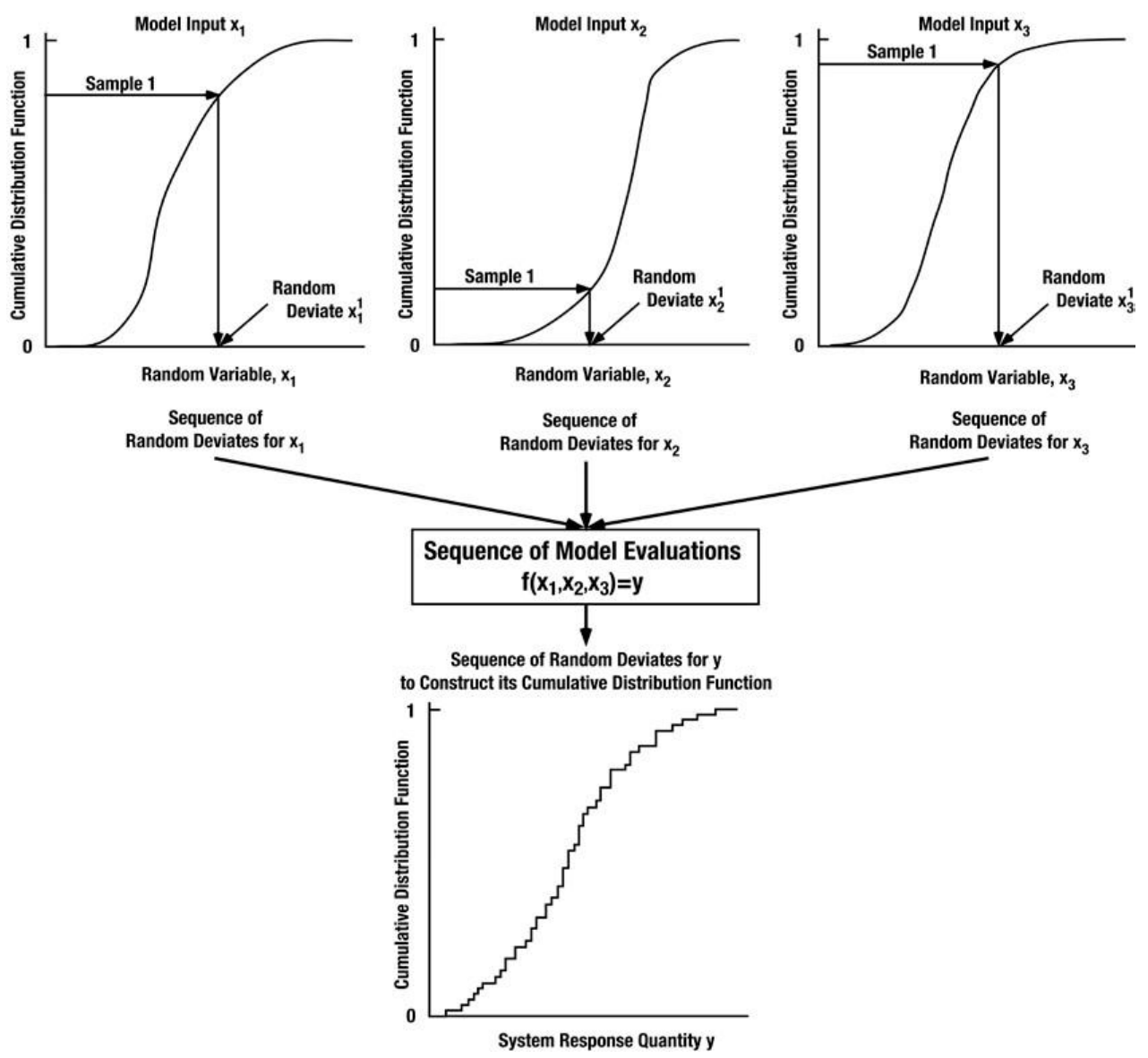

Figure 9. Monte Carlo sampling to propagate aleatory uncertainties through a model (from [3]).

A more advanced approach for propagating aleatory uncertainty through the model is to use polynomial chaos [33]. Polynomial chaos methods approximate both stochastic model inputs and output (i.e., SRQ) using series expansions to replace stochastic equations by deterministic systems that are then finitely truncated and solved discretely. While initial polynomial chaos implementations were code intrusive, more recently non-intrusive forms of polynomial chaos have become popular. When only a few aleatory uncertain variables are present (e.g., less than ten), then polynomial chaos can significantly reduce the number of samples required for statistical convergence of the CDF. However, the method has a notable limitation in that for large number of random variables, polynomial chaos becomes very computationally expensive and traditional sampling methods are typically more feasible.

\subsubsection{Combined Aleatory and Epistemic Uncertainty}

When aleatory and epistemic uncertainties occur in the input quantities, the sampling for each type of uncertainty must be separated. As mentioned above, each of the samples obtained from an aleatory uncertainty is associated with a probability of occurrence. When a sample is taken from an epistemic uncertainty, however, there is no probability associated with the sample. The sample is simply a possible realization over the interval-valued range of the input quantity. For example, if one takes a sample from each of the epistemic uncertainties, and then one 
computes the aleatory uncertainty as just described, the computed CDF of the SRQ can be viewed as a conditional probability. That is, the computed CDF is for the condition of the given vector of fixed samples of the epistemic uncertainties. This type of segregated sampling between aleatory and epistemic uncertainties is usually referred to as double-loop or nested sampling.

For epistemic uncertainties, Latin hypercube sampling (LHS) is recommended [34, 35]. For LHS over a single uncertain input, the probabilities are separated into a number of equally-sized divisions and one sample is randomly chosen in each division. Since there is absolutely no structure over the range of the interval of the epistemic uncertainty, an appropriate structure for sampling would be a combinatorial design. The number of samples, $M$, of the epistemic uncertainties must be sufficiently large to insure satisfactory coverage of the combinations of all of the epistemic uncertainties in the mapping to the SRQs. Based on the work of Refs. [35 - 37], we recommend that a minimum of three LHS samples be taken for each epistemic uncertainty, in combination with all of the remaining epistemic uncertainties [3]. For example, if $m$ is the number of epistemic uncertainties, the minimum number of samples would increase as $\mathrm{m}^{3}$. Recall that for each of these combinations, one must compute all of the samples for the aleatory uncertainties. For more than about four or five epistemic uncertainties, the total number of samples required for convergence becomes extraordinarily large.

For each sample of all of the epistemic uncertainties, combined with all of the probabilistic samples for the aleatory uncertainties, a single CDF of the SRQ will be produced. After all of the epistemic and aleatory samples have been computed, one has an ensemble of $M$ CDFs. The widest extent of the ensemble of CDFs is used to form a p-box [16, $22,23]$. The probability box is a special type of CDF that contains information on both aleatory and epistemic uncertainties (see Figure 10). A probability box expresses both epistemic and aleatory uncertainty in a way that does not confound the two. A probability box shows that an SRQ cannot be displayed as a precise probability, but it is now an interval-valued probability. For example, in Figure 10, for a given value of the SRQ, the probability that that value will occur is given by an interval-valued probability. That is, no single value of probability can describe the uncertainty, given the present state of knowledge. Likewise, for a given probability value of the SRQ, there is an interval-valued range for the SRQ of interest. Stated differently, the probability box accurately reflects the system response given the state of knowledge of the input uncertainties.

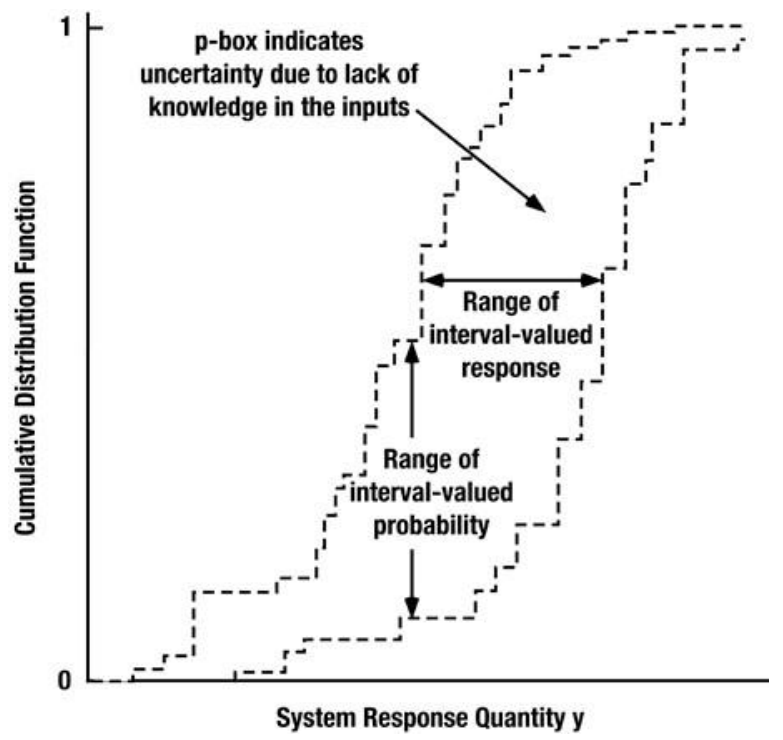

Figure 10. Sampling to propagate combined aleatory and epistemic uncertainties through a model (from [3]).

\subsubsection{Estimate Model Form Uncertainty}

Model form uncertainty is due to aggregation of all assumptions and approximations in the formulation of the model [3]. Model form uncertainty is estimated through the process of model validation. As mentioned above, there are two aspects to estimating model form uncertainty. First, we quantitatively estimate the model form uncertainty at the 
conditions where experimental data are available (i.e., within the validation domain) using a mathematical operator referred to as a validation metric. During the last ten years there has been a flurry of activity dealing with the construction of validation metrics $[5,16,38-40]$. Second, we extrapolate the error structure expressed by the validation metric to the application conditions of interest (i.e., over the application domain). As is common in scientific computing, no experimental data is available for the application conditions of interest. Then the extrapolated model form uncertainty is included in the prediction of the model at the conditions of interest as an epistemic uncertainty. This section discusses both of these topics.

\subsubsection{Validation Metrics}

Validation metrics provide a means by which the accuracy of a model can be assessed relative to experimental observations by providing measures of the agreement/disagreement between the model and experimental data [3]. The field of validation metrics is currently an area of active research with a number of different methods and approaches, but for the purposes of this section, we limit our discussion to the description and comparison of two of the leading approaches for assessing and estimating model form uncertainty: 1) Bayesian validation methods and 2) stochastic validation metrics.

Although we previously referred to parameter estimation and model updating as model calibration, many researchers and practitioners in the field refer to these approaches using model updating techniques as model validation. Often in science and engineering applications there are parameters within a model whose values are not known from theory or prior measurements. The best values - or a multivariate probability distribution - for these parameters for a given application must be inferred from new measurements. This inference process is calibration. In practice, the problem of model calibration is often formulated as finding the parameters that minimize the squared difference between the model-computed data (the predicted data) and the actual experimental data. As such, whereas in uncertainty propagation we are interested in the forward propagation of uncertainty, in model updating, we are interested in the inverse problem of uncertainty analysis, in which experimental data are used to calibrate the parameters of a computer model. This approach, however, does not allow for explicit treatment of uncertainty or error in the model itself: the model is considered the "true" deterministic representation of reality. In other words, updating assumes that the model itself is correct. While this approach does have utility, it is far from an accurate mathematical treatment of the true model calibration problem in which both the computed data and experimental data have error bars.

More recently, research in the Bayesian statistics community has yielded advances in formal statistical methods that address calibration under uncertainty. While calibration is still used to tune unknown calibration parameters of the computer model, the addition of a discrepancy function has been used to capture model discrepancy (also termed model bias or model inadequacy) due to underlying missing physics, numerical approximations, and other inaccuracies of the computer model that would exist even if all calibration parameters are known. In particular, these methods formulate a model for calibration data that includes an experimental error term (similar to standard regression) and a model discrepancy term, with a Gaussian process chosen to model the discrepancy. Bayesian approaches [42 - 51] are then used to update the statistical parameters associated with the discrepancy term and with the model parameters. The purpose of updating is generally to reduce uncertainty in the parameters through the application of additional information (e.g., new experimental data). The expectation is that the reduced uncertainty increases the predictive content of the calibration.

The Bayesian updating approach in the context of predictive modeling and simulation is presented in Figure 3 in which a general flowchart for model updating (calibration), validation, and prediction processes are shown. The goal of model updating is to create an updated model that integrates the computer model simulations and experimental data to better predict in regions where experimental data have not been collected. We denote the controllable inputs (i.e., design variables) by the vector $\mathrm{X}=\left[x_{1}, \ldots, x_{d}\right]^{T}$ and the calibration parameters, which are unknown model parameters, by the vector $\theta=\left[\theta_{1}, \ldots, \theta_{r}\right]^{T}$. A calibration parameter is defined as any physical parameter that can be specified as an input to the computer model and that is unknown or not measureable when conducting the physical experiments [46]. The iterative model updating process begins by simulating the computer model response, $y^{m}(\mathrm{X}, \Theta)$, at a set of $N_{m}$ input sites $\mathrm{X}^{m}=\left[\mathrm{x}_{1}^{m}, \ldots, \mathrm{x}_{N_{m}}^{m}\right]^{T}$ and corresponding calibration parameter values $\Theta^{m}=\left[\Theta_{1}^{m}, \ldots, \Theta_{N_{m}}^{m}\right]^{T}$, where each $\left\{\mathrm{x}_{i}^{m}, \Theta_{i}^{m}\right\}$ is a combination of input and calibration parameter settings at which the simulation is run. One then observes the physical experimental response, $y^{e}(\mathrm{x})$, at a set of $N_{e}$ input sites 
$\mathrm{X}^{e}=\left[\mathrm{x}_{1}^{e}, \ldots, \mathrm{x}_{N_{e}}^{e}\right]^{T}$, often using design of experiments methods $[19-21]$ to select the input sites. After collecting the data, an updated model is created using various formulations and methods of inference. There are three possible Bayesian model updating formulations: 1) discrepancy function with no calibration parameters, 2) calibration parameters with no discrepancy function, and 3) calibration parameters with discrepancy function.

The first model updating formulation considers a discrepancy function with no calibration parameters. A Bayesian approach is considered in references [42,55], which model the system as

$$
y^{e}(\mathrm{x})=y^{m}(\mathrm{x})+\delta(\mathrm{x})+\varepsilon
$$

where $y^{e}(\mathbf{x})$ is the experimental response, $y^{m}(\mathbf{x})$ denotes the computer model response, $\delta(\mathbf{x})$ is the additive discrepancy function, and $\varepsilon$ accounts for the experimental uncertainty. Experimental uncertainty is typically assumed to follow a normal distribution with mean 0 and variance $\lambda$, denoted $\varepsilon \sim N(0, \lambda)$. The advantage of considering only a discrepancy function is that a Bayesian closed form solution exists, since the discrepancy function is nearly directly observable from the collected simulations and experimental data. The obvious limitation of the formulation in Equation 2 is its inability to account for the effects of unknown calibration parameters.

A second formulation incorporates calibration parameters with no discrepancy function. This formulation assumes the model

$$
y^{e}(\mathrm{x})=y^{m}\left(\mathrm{x}, \theta^{*}\right)+\varepsilon
$$

where $y^{m}(\mathbf{x}, \theta)$ is the computer model response as a function of $\mathbf{x}$ and $\theta$, and $\theta^{*}$ (a $r \times 1$ vector of constants) denotes the true values of the unknown calibration parameters over the course of the physical experiments. Various methods, such as nonlinear least squares, can be used to estimate $\theta^{*}$ [53], although this is not trivial if the input (x) values are not the same over the physical experimental and simulation runs. For this reason, many instances of this approach [54] only consider calibration parameters and omit $\mathbf{x}$ when fitting the model.

Figure 11(a) shows a hypothetical example of $y^{m}\left(x, \theta^{*}\right)$ for different values of $\theta$, together with $y^{e}(x)$ with no experimental uncertainty or model discrepancy (i.e., $y^{m}\left(x, \theta^{*}\right)$ ). With no model discrepancy, there exists a value of $\theta$ such that the computer model response is in perfect agreement with the physical response, and this value is precisely $\theta^{*}$. In contrast, for the simple case of $\varepsilon=0$, Figure 11(b) illustrates the problem with not including a discrepancy function in the model when model discrepancy truly exists. In this case, there is no value of $\theta$ for which the computer model response will be in perfect agreement with the experimental response. However, inclusion of a discrepancy function will allow the model of Equation (3) to agree with the experimental response.

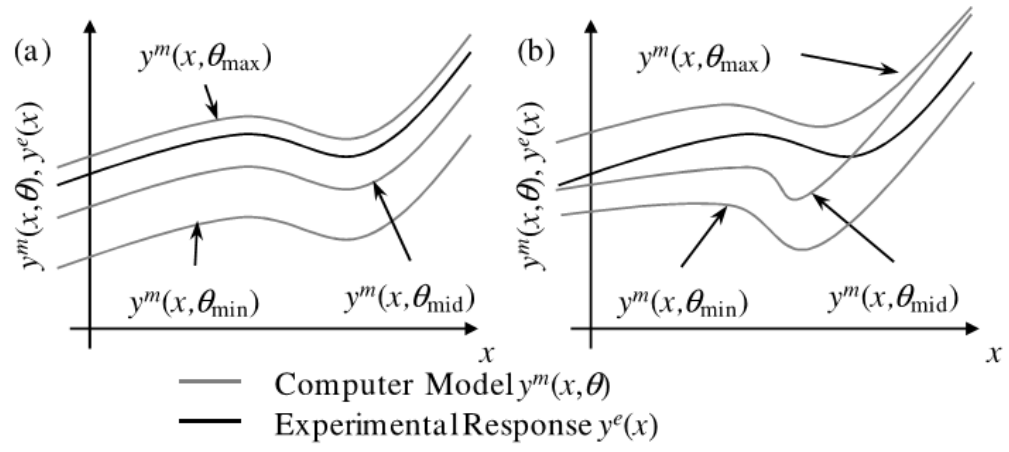

Figure 11. Example of a response considering a calibration parameter (a) without and (b) with the need for a discrepancy function (from [61]).

The third formulation $[46,48,56]$ combines the two approaches above into one model updating formulation represented by

$$
y^{e}(\mathrm{x})=y^{m}(\mathrm{x}, \theta)+\delta(\mathrm{x})+\varepsilon
$$


The formulation in Equation 4 addresses parameter uncertainty, model discrepancy, experimental uncertainty, and interpolation uncertainty. A quantification of the latter is a by-product of the Gaussian process metamodeling. To quantify uncertainty in the calibration parameters and discrepancy function, the modular Bayesian approach is used to calculate their posterior distributions. Notice that the discrepancy function in Equation 4 is not directly observable from the collected data, since the true value of $\theta$ is unknown. Because Equation 4 accounts for several different forms of uncertainty and also includes $\mathbf{x}$, one can view it as providing a comprehensive and widely applicable model updating formulation for calibration under uncertainty.

The majority of the approaches for model updating consider either calibration only [53,54] or discrepancy function only [42, 55], and few consider both calibration and a discrepancy function [46, 48, 52, 56, 58, 59 - 60] together. The model updating formulation of Kennedy and O'Hagan [46] is one such approach that incorporates both calibration parameters and a discrepancy function, and we believe this to be the most applicable approach for calibration under uncertainty and for assessing and estimating model form uncertainty. However, it is noted that one of the main challenges in model updating is the difficulty in distinguishing between the effects of calibration parameters versus model discrepancy. This issue or effect is known as a lack of identifiability of calibration parameters in conjunction with the model discrepancy. Identifiability refers to whether the single true value of a model's calibration parameters can theoretically be inferred [45] based on the available data.

Most of the existing model updating techniques $[46,48,52,56]$ treat the process as a black-box and have the objective of improving the experimental response prediction but with little regard to identifying the true model calibration parameters and model discrepancy. Loeppky et al. [58] explored the identifiability issue and concluded that accurate prediction of the experimental response is often possible, even if the individual calibration parameters and discrepancy function are not identified precisely. Furthermore, they concluded that the true values of the calibration parameters are only attainable when the model discrepancy between simulation and reality is neither present nor included in the model updating formulation. Higdon et al. [56] found that in some simple cases the calibration parameters and discrepancy function can be accurately identified, but in other cases their effects cannot be distinguished even though the response predictions may be reasonable.

In discussing model updating, we also wish to emphasize the difference between calibration and validation. Calibration of a computational model is adjusting a set of model parameters associated so that we maximize the model agreement with a set of experimental data (or, in certain cases, a set of numerical benchmarks). Validation of a computational model is quantifying our belief in the predictive capability of a computational model through comparison with a set of experimental data. Uncertainty in both the data and the model is critical and must be mathematically understood to do both calibration and validation correctly. Therefore, to assess the predictive capability of a model, one would have to conduct model validation as a follow-up process that determines whether the updated model accurately reflects reality based on additional experimental response data and various validation metrics [3]. As shown in Figure 3, if the validation assessment indicates that the model is inadequate, one can either collect more data to further update the model or refine/revise the computer model by changing its physics.

An alternative to Bayesian model updating methods for assessing and estimating model form uncertainty is the use of stochastic validation metrics. While there are many possible stochastic validation metrics, in this paper and in the AIAA Guide Update, we will focus on one implementation called the area validation metric [16], which is a mathematical metric that provides quantitative assessment of disagreement between a stochastic model output and experimental measurements of the same system response quantity. When only aleatory uncertainties are present in the model inputs, then propagating these uncertainties through the model produces a CDF of the SRQ. Experimental measurements are then used to construct an empirical CDF of the SRQ. The area between these two CDFs is referred to as the area validation metric $d$ (also called the Minkowski $\mathrm{L}_{1}$ norm) and is given by

$$
d(F, S n)=\int_{-\infty}^{\infty}|F(x)-\operatorname{Sn}(x)| d x
$$

where $F(x)$ is the CDF from the simulation, $\operatorname{Sn}(x)$ is the CDF from the experiment, and $x$ is the SRQ. The area validation metric $d$ has the same units as the SRQ and thus provides a measure of the evidence for disagreement between the simulation and the experiment [16]. Note that the area validation metric represents an epistemic uncertainty because it embodies the bias effect of all of the assumptions and approximations in the formulation of the mathematical model, as compared to measurements of the SRQ in nature. Note that the area validation metric 
can also contain sampling (epistemic) uncertainty due to a finite number of experimental measurements, or a finite number of computational samples. The area validation metric attempts to capture the intrinsic modeling uncertainty and is commonly referred to as model form uncertainty.

An example of this area validation metric for a case with only aleatory uncertainties occurring in the model input parameters is given in Figure 12. In this figure, the aleatory uncertainties have been propagated through the model (e.g., with a large number of Monte Carlo samples), but only four experimental replicate measurements are available. The stair-steps in the experimental CDF are due to the different values observed in each of the four experimental measurements. The stochastic nature of the measurements can be due to variability of the experimental conditions and random measurement uncertainty. This metric can also be computed for cases involving both aleatory and epistemic uncertainty in the model inputs (e.g., see Ref. [3]).

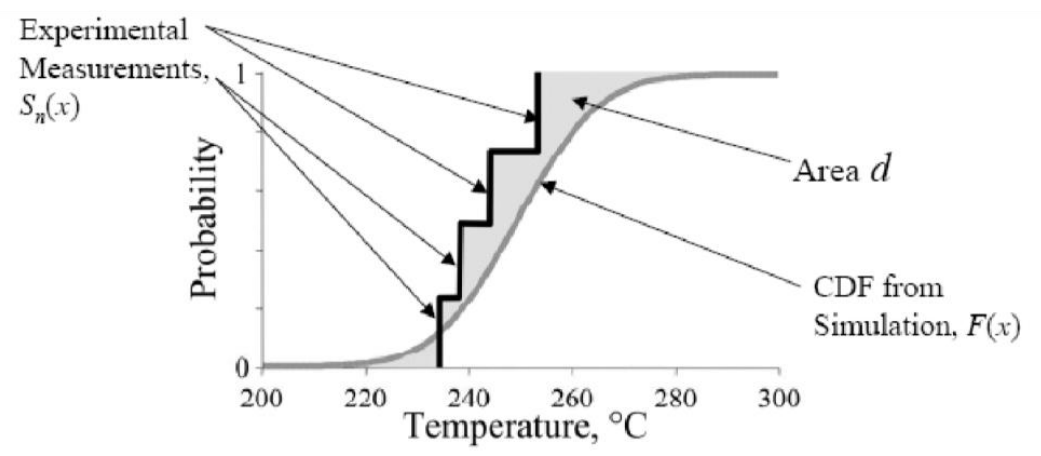

Figure 12. Area validation metric example with four experimental measurements of the SRQ available (from [16]).

Figure 13 is an example of the area metric for the case when there are only three samples from the simulation and five experimental measurements. This situation is not uncommon for computationally intensive scientific computing applications such as computational fluid dynamics. A significant advantage of the area validation metric is that it can be computed even when very few samples are available from the simulation and from the experiment. In addition, when only very few simulation or experimental samples are available, no assumptions are required concerning the statistical nature of the simulation or the experiment. The validation metric deals directly with the samples computed and the measurements obtained.

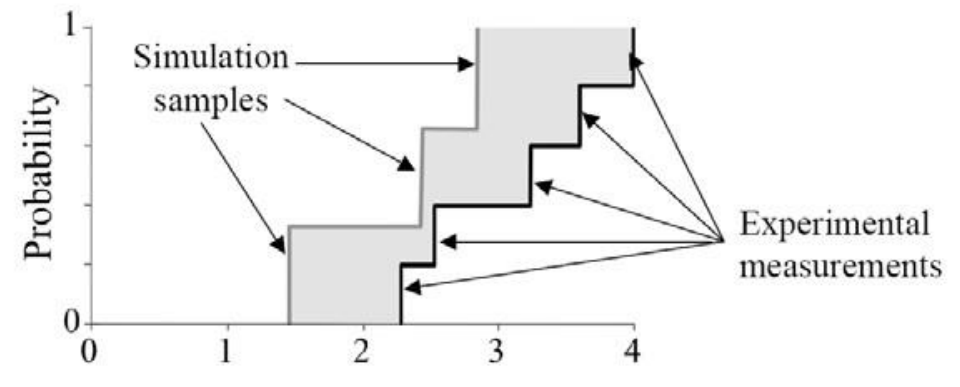

Figure 13. Example of the area validation metric when only a small number of simulations and experimental measurements are available (from [16]).

When little experimental information is available on needed model input parameters, the parameters are characterized as an interval, i.e., epistemic uncertainties. This situation occurs very commonly with published experimental data and experiments that were not designed to be validation experiments. As a result, when these intervals are propagated through the model, the predicted SRQ of interest is represented as a probability box. The validation metric can also deal with this situation. Figure 14 shows the case where the model prediction is a probability box and only a single experimental measurement is available. Figure 14a occurs when the measurement falls entirely within the probability box and Figure 14b occurs when the measurement is slightly larger than the probability box. When the experimental measurement falls entirely within the simulation's probability box (Figure 
14a), then the area validation metric is zero. When a portion of the experimental measurement falls outside of the probability box (Figure 14b), then the area validation metric is nonzero. Recall that the area validation metric is the smallest area between the probability box and the experimental CDF. That is, the validation metric reflects the evidence for disagreement between the model and the experiment. When the simulation is a probability box due to insufficient information provided by the validation experiment, the model is given more leeway in comparing with the experiment, as is appropriate.

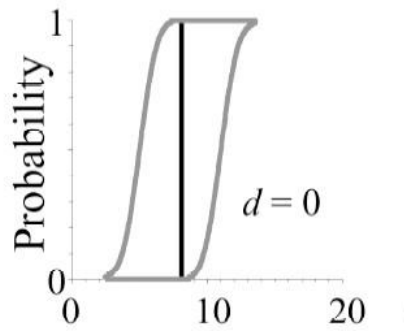

a)

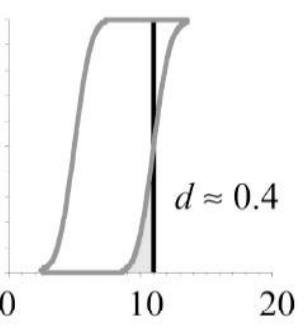

b)

Figure 14. Example of area validation metric computed at one location in the model input parameter space: aleatory uncertainties only (left) and combined aleatory and epistemic uncertainties (right) (from [16]).

\subsubsection{Model Extrapolation}

Numerous validation experiments would be required to estimate the validation metric over the entire space of model input parameters for the application of interest. In many cases, however, it is not even possible to obtain experimental data at the application conditions of interest. As a result, the more common case is that the model must be applied at conditions where there are no experimental data. Consider a simple example when there are only two input parameters for the model: $\alpha$ and $\beta$ (Figure 15). The validation domain consists of the set of points in this parameter space where experiments have been conducted and the validation metric has been computed (denoted by a "V" in Figure 15). The application domain, sometimes referred to as the operating envelope of the system, is generally larger than the validation domain. Thus, one must choose between either extrapolating the validation metric outside of the validation domain or performing additional validation experiments. In Figure 15, conditions for candidate validation experiments are given by a " $\mathrm{C}$ ".

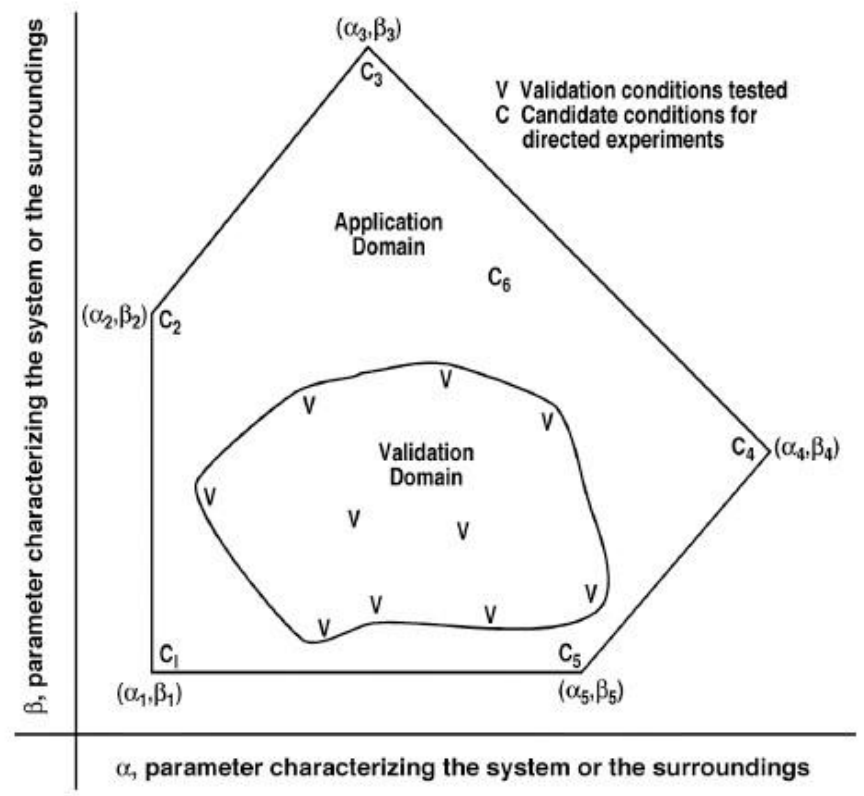

Figure 15. Model validation relative to experimental data (from [3]). 
In the past, it has been common practice to either a) ignore the model form uncertainty in the predictions for the application conditions or b) calibrate and update adjustable parameters in the mathematical model so that improved agreement could be obtained with the available experimental data at conditions "V". We, however, believe a more forthright approach is to explicitly account for the mismatch between the model and the experiments and include this mismatch as additional uncertainty in the predictions of the SRQ in the application domain. When the validation metric results are extrapolated to new conditions, there is a subsequent increase in the uncertainty due to the extrapolation process itself. It should be stressed that this extrapolation is a statistical extrapolation of the uncertainty structure of the validation metric that has been observed over the validation domain. The extrapolation of the uncertainty structure is completely separate from any extrapolation of the simulation prediction results to conditions where experimental data are not available. A common example of the latter is the use of an aerodynamics model to extrapolate from wind tunnel conditions (where data exist) to flight Reynolds numbers.

It is noted that model extrapolation can occur in terms of input parameters and extrapolation to higher levels in the validation hierarchy. Large extrapolations may commonly involve large changes in coupled physics, e.g., heating effects on structural dynamics, and large changes in geometry or subsystem interactions, e.g., abnormal or hostile environments. It is our firm belief that large extrapolations must be based on physics inference, not statistical inference as there is no rigorous mathematical basis for quantifying the uncertainty in computationally estimated SRQ of interest. Above all, large extrapolations should result in large increase in uncertainty.

\subsubsection{Determine Total Uncertainty in the SRQ}

The total prediction uncertainty can be estimated by combining the propagated uncertainty from the model inputs (aleatory and epistemic) with the uncertainty due to the form of the model and the uncertainty due to the numerical error estimation process. In the described imprecise probability approach to uncertainty quantification, the total uncertainty in the SRQ at the application conditions of interest is computed as follows. First, one begins with the probability box that was generated by propagating the aleatory and epistemic uncertainties in the model input parameters through the model (recall Figure 8 and Figure 10). Next, the area validation metric is appended to the sides of the probability box, thus showing that the epistemic uncertainty in the SRQ has increased due to model form uncertainty. As discussed above, if extrapolation of the model form uncertainty is required, then it is the extrapolated $d$ values that are appended to the SRQ probability box. This process is shown graphically in Figure 16.

Finally, the uncertainty due to numerical approximations is treated as an additional epistemic uncertainty and, in a similar fashion as the model form uncertainty, it is appended to the probability box of the SRQ.

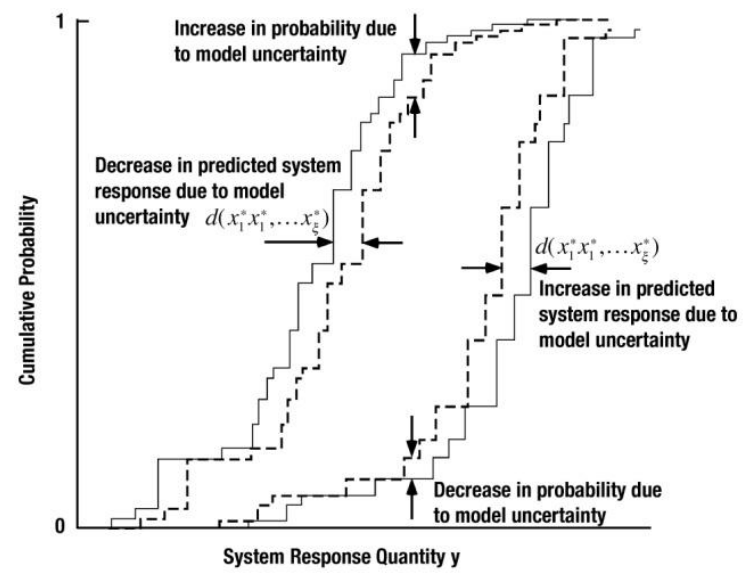

Figure 16. Increase in predictive uncertainty due to the addition of model form uncertainty (from [3]).

This approach for representing total uncertainty in the predicted SRQ of interest provides valuable information to decision makers using the results from the simulations. The width of the original probability box provides information on the effects of epistemic uncertainties in the model inputs on the predicted SRQ. The shape, or the range, of the two bounding CDFs of the probability box provides information on the effects of aleatory uncertainties in the model inputs. The validation metric $d$ that is appended to the probability box of the SRQ tells the decision maker the magnitude of the uncertainty that is due to model form uncertainty. Finally, $U_{N U M}$ that is appended to the 
probability box informs the decision maker of the magnitude of the uncertainty due to numerical approximations. In limited testing of this approach, it has been found that the contribution of model form uncertainty has been a dominant contributor to the uncertainty in the predicted SRQ, even for relatively accurate models of the system of interest $[3,16]$.

\section{Conclusions}

The framework for verification, validation, and uncertainty quantification in scientific computing presented here and to be included in the upcoming AIAA Guide represents a conceptual shift in the way that scientific and engineering predictions are presented to decision makers. The philosophy of the present approach is to rigorously segregate aleatory and epistemic uncertainties in input quantities, and explicitly account for numerical approximation errors and model form uncertainty directly in terms of the predicted quantities of interest. In this way, the decision maker is clearly and unambiguously shown the uncertainty in the predicted system response. For example, if the model has been found to be relatively inaccurate in previous comparisons with experimental data, the decision maker will starkly see this in any new predictions; as opposed to a newly calibrated model based on recent experiments. We believe this philosophy of presenting predictions to decision makers is needed to reduce the often seen failure in under estimating predictive uncertainty. We believe that with this clearer picture of the uncertainties, the decision maker is better served. This approach is particularly important for predictions of high-consequence systems, such as those where human life, the public safety, national security, or the future of a company is at stake.

\section{References}

1. AIAA, "Guide for the Verification and Validation of Computational Fluid Dynamics Simulations," American Institute of Aeronautics and Astronautics, AIAA-G-077-1998, Reston, VA, 1998.

2. ASME, "Guide for Verification and Validation in Computational Solid Mechanics," American Society of Mechanical Engineers, ASME Standard V\&V 10-2006, New York, NY, 2006.

3. Oberkampf, W. L. and Roy, C. J., Verification and Validation in Scientific Computing, Cambridge University Press, New York, 2010.

4. Oberkampf, W. L. and Trucano, T. G., "Verification and Validation Benchmarks," Nuclear Engineering and Design, 238(3), 2008, pp. $716-743$.

5. Oberkampf, W. L. and Barone, M. F., "Measures of Agreement between Computation and Experiment: Validation Metrics," Journal of Computational Physics, Vol. 217, No. 1, 2006, pp. 5 - 36.

6. Oberkampf, W. L., Trucano, T. G., and Hirsch, C., "Verification, Validation, and Predictive Capability," Applied Mechanics Reviews, Vol. 57, No. 5, 2004, pp. 345 - 384.

7. Morgan, M. G. and Henrion, M., Uncertainty: A Guide to Dealing with Uncertainty in Quantitative Risk and Policy Analysis, $1^{\text {st }}$ Ed., Cambridge University Press, Cambridge, UK, 1990.

8. Cullen, A. C. and Frey, H. C., Probabilistic Techniques in Exposure Assessment: A Handbook for Dealing with Variability and Uncertainty in Models and Inputs, Plenum Press, New York, 1999.

9. Vose, D., Risk Analysis: A Quantitative Guide, $3^{\text {rd }}$ Ed., Wiley, New York, 2008.

10. Haimes, Y. Y., Risk Modeling, Assessment, and Management, $3^{\text {rd }}$ Ed., John Wiley, New York, 2009.

11. Bedford, T. and Cooke R., Probabilistic Risk Analysis: Foundations and Methods, Cambridge University Press, Cambridge, UK, 2001.

12. Ghosh, J. K., Delampady, M., and Samanta, T., An Introduction to Bayesian Analysis: Theory and Methods, Springer, Berlin, 2006.

13. Sivia, D. and Skilling, J., Data Analysis: A Bayesian Tutorial. $2^{\text {nd }}$ Ed., Oxford University Press, Oxford, 2006.

14. Coleman, H. W. and Stern F., "Uncertainties and CFD Code Validation," Journal of Fluids Engineering. Vol. 119, 1997, pp. $795-803$.

15. Stern, F., Wilson, R. V., Coleman H. W., and Paterson E. G., "Comprehensive Approach to Verification and Validation of CFD Simulations-Part 1: Methodology and Procedures," Journal of Fluids Engineering, Vol. 123, No. 4, 2001, pp. $793-802$. 
16. Ferson, S., Oberkampf, W. L., and Ginzburg, L., "Model Validation and Predictive Capability for the Thermal Challenge Problem," Computer Methods in Applied Mechanics and Engineering. Vol. 197, 2008, pp. 2408 2430 .

17. Coleman, H. W. and Steele, W. G., Experimentation, Validation, and Uncertainty Analysis for Engineers, Wiley, New York, 1999.

18. Youden, W. J., "Enduring Values," Technometrics, Vol. 14, No. 1, 1972.

19. Montgomery, D. C., Design and Analysis of Experiments, $5^{\text {th }}$ Ed., John Wiley, Hoboken, NJ, 2000.

20. Box, G. E. P., Hunter, J. S., and Hunter, W. G., Statistics for Experimenters: Design, Innovation, and Discovery, $2^{\text {nd }}$ Ed., John Wiley, New York, 2005.

21. Oberkampf, W. L. and Aeschliman D. P., "Joint Computational/Experimental Aerodynamics Research on a Hypersonic Vehicle: Part 1, Experimental Results,” AIAA Journal, Vol. 30, No. 8, 1992, pp. 2000 - 2009.

22. Ferson, S. and Ginzburg L. R., "Different Methods are Needed to Propagate Ignorance and Variability," Reliability Engineering and System Safety, Vol. 54, 1996, pp. 133 - 144.

23. Ferson, S. and Hajagos J. G., "Arithmetic with Uncertain Numbers: Rigorous and (Often) Best Possible Answers," Reliability Engineering and System Safety. Vol. 85, Nos. 1-3, 2004, pp. 135 - 152.

24. Roache, P. J., Verification and Validation in Computational Science and Engineering, Hermosa Publishers, New Mexico, 1998.

25. Cavallo, P. A. and Sinha, N., "Error Quantification for Computational Aerodynamics Using an Error Transport Equation," Journal of Aircraft, Vol. 44, No. 6, 2007, pp. 1954 - 1963.

26. Shih, T. I-P. and Williams, B. R., "Development and Evaluation of an A Posteriori Method for Estimating and Correcting Grid-Induced Errors in Solutions of the Navier-Stokes Equations," AIAA Paper 2009-1499, 2009.

27. Ainsworth, M. and Oden, J. T., "A Posteriori Error Estimation in Finite Element Analysis," Computer Methods in Applied Mechanics and Engineering, Vol. 142, No. 1-2, 1997, pp. $1-88$.

28. Ainsworth, M. and Oden, J. T., A Posteriori Error Estimation in Finite Element Analysis, Wiley Interscience, New York, 2000.

29. Zienkiewicz, O. C. and Zhu, J. Z., "The Superconvergent Patch Recovery and A Posteriori Error Estimates, Part 2: Error Estimates and Adaptivity," International Journal for Numerical Methods in Engineering, Vol. 33, 1992, pp. $1365-1382$.

30. Roy, C. J., "Review of Discretization Error Estimators in Scientific Computing," AIAA Paper 2010-0126, 2010, Orlando, FL.

31. Roy, C. J., "Review of Code and Solution Verification Procedures for Computational Simulation," Journal of Computational Physics, Vol. 205, No. 1, 2005, pp. 131 - 156.

32. Roache, P. J., "Perspective: A Method for Uniform Reporting of Grid Refinement Studies," Journal of Fluids Engineering, Vol. 116, 1994, pp. 405 - 413.

33. Ghanem, R. and Spanos, P., Stochastic Finite Elements: A Spectral Approach, Springer Verlag, 1991.

34. Helton, J. C., "Uncertainty and Sensitivity Analysis in the Presence of Stochastic and Subjective Uncertainty," Journal of Statistical Computation and Simulation, Vol. 57, 1997, pp. 3 - 76.

35. Sallaberry, C. J., Helton, J. C., and Hora, S. C., "Extension of Latin Hypercube Samples with Correlated Variables," Reliability Engineering and System Safety, Vol. 93, 2008, pp. 1047 - 1059.

36. Ferson, S. and Tucker W. T., "Sensitivity in Risk Analyses with Uncertain Numbers," Sandia National Laboratories, SAND2006-2801, Albuquerque, NM, 2006.

37. Kreinovich, V., Beck, J., Ferregut, C., Sanchez, A., Keller, G.R., Averill M., and Starks, S.A., "Monte-CarloType Techniques for Processing Interval Uncertainty, and Their Potential Engineering Applications," Reliable Computing. Vol. 13, 2007, pp. $25-69$.

38. Coleman, H. W. and Stern, F., "Uncertainties and CFD Code Validation," Journal of Fluids Engineering, Vol. 119, 1997, pp. $795-803$.

39. Stern, F., Wilson, R. V., Coleman, H. W., and Paterson, E. G., "Comprehensive Approach to Verification and Validation of CFD Simulations-Part 1: Methodology and Procedures," Journal of Fluids Engineering, Vol. 123, No. 4, 2001, pp. $793-802$.

40. Oberkampf, W. L. and Trucano, T. G., "Verification and Validation in Computational Fluid Dynamics," Progress in Aerospace Sciences, Vol. 38, No. 3, 2002, pp. 209 - 272.

41. Roy, C. J. and Oberkampf, W. L., "A Comprehensive Framework for Verification, Validation, and Uncertainty Quantification in Scientific Computing," Computer Methods in Applied Mechanics and Engineering, Vol. 200, Issues 25-28, 2011, pp. $2131-2144$.

42. Chen, W., Xiong, Y., Tsui, K. L., and Wang, S., "A Design-driven Validation Approach using Bayesian Prediction Models," Journal of Mechanical Design, Vol. 130, Issue 2, 2008, pp. 021101. 
43. Anderson, M. C., Hasselman, T. K., and Carne, T. G., "Model Correlation and Updating of a Nonlinear Finite Element Model using Crush Test Data," $17^{\text {th }}$ International Modal Analysis Conference (IMAC) on Modal Analysis, Paper No. 376, Kissimmee, FL, Proceedings of the Society of Photo-Optical Instrumentation Engineers, 1999, pp. 1511 - 1517.

44. Hanson, K. M., “A Framework for Assessing Uncertainties in Simulation Predictions,” Physica D, Vol. 133, 1999, pp. $179-188$.

45. Lancaster, T., An introduction to modern Bayesian econometrics, Blackwell Publishing, Malden, MA, 2004.

46. Kennedy, M. C., and O'Hagan, A., "Bayesian Calibration of Computer Models," Journal of the Royal Statistical Society: Series B, Vol. 63, Issue 3, 2001, pp. 425 - 464.

47. Hasselman, T. K., Wathugala, G. W., and Crawford, J., "A Hierarchical Approach for Model Validation and Uncertainty Quantification," Fifth World Congress on Computational Mechanics, Vienna, Austria, Vienna University of Technology, 2002.

48. Bayarri, M. J., Berger, J. O., Paulo, R., Sacks, J., Cafeo, J. A., Cavendish, J., Lin, C. H., and Tu, J., “A Framework for Validation of Computer Models," Technometrics, Vol. 49, Issue 2, 2007, pp. 138 - 154.

49. O'Hagan, A., "Bayesian Analysis of Computer Code Outputs: A Tutorial," Reliability Engineering and System Safety, Vol. 91, Issues $10-11,2006$, pp. $1290-1300$.

50. Trucano, T. G., Swiler, L. P., Igusa, T., Oberkampf, W. L., and Pilch, M., "Calibration, Validation, and Sensitivity Analysis: What's What," Reliability Engineering and System Safety, Vol. 91, Issues 10 - 11, 2006, pp. $1331-1357$.

51. Babuska, I., Nobile, F., and Tempone, R., "A Systematic Approach to Model Validation based on Bayesian Updates and Prediction Related Rejection Criteria," Computer Methods in Applied Mechanics and Engineering, Vol. 197, Issues 29 - 32, 2008, pp. 2517 - 2539.

52. Xiong, Y., Chen, W., Tsui, K. L., and Apley, D. W., “A Better Understanding of Model Updating Strategies in Validating Engineering Models," Computer Methods in Applied Mechanics and Engineering, Vol. 198, Issues 15 - 16, 2009, pp. $1327-1337$.

53. Lindgren, L. E., Alberg, H., and Domkin, K., "Constitutive Modelling and Parameter Optimization," 7th International Conference on Computational Plasticity, Barcelona, Spain, 2003.

54. McFarland, J., Mahadevan, S., Romero, V., and Swiler, L., "Calibration and Uncertainty Analysis for Computer Simulations with Multivariate Output," AIAA Journal, Vol. 46, Issue 5, 2008, pp. 1253 - 1265.

55. Wang, S., Tsui, K. L., and Chen, W., "Bayesian Validation of Computer Models," Technometrics, Vol. 51, Issue 4, 2009, pp. $439-451$.

56. Higdon, D., Kennedy, M. C., Cavendish, J., Cafeo, J., and Ryne, R., "Combining Field Data and Computer Simulations for Calibration and Prediction," SIAM Journal on Scientific Computing, Vol. 26, Issue 2, 2004 , pp. $448-466$.

57. Higdon, D., Nakhleh, C., Gattiker, J., and Williams, B., "A Bayesian Calibration Approach to the Thermal Problem," Computer Methods in Applied Mechanics and Engineering, Vol. 197, 2010, pp. 2431 - 2441.

58. Loeppky, J., Bingham, D., and Welch, W., "Computer Model Calibration or Tuning in Practice," University of British Columbia, Research Technical Report, Vancouver, BC, CA, 2006.

59. Rougier, J., "Probabilistic Inference for Future Climate Using an Ensemble of Climate Model Evaluations," Climate Change, Vol. 81, Issues 3-4, 2007, pp. $247-264$.

60. Liu, F., Bayarri, M. J., Berger, J. O., Paulo, R., and Sacks, J., "A Bayesian Analysis of the Thermal Challenge Problems," Computer Methods in Applied Mechanics and Engineering, Vol. 197, Issues 29 - 32, 2009, pp. 2457 $-2466$.

61. Arendt, P., Apley, D., and Chen, W., "Quantification of Model Uncertainty: Calibration, Model Discrepancy, and Identifiability,” Journal of Mechanical Design, Vol. 134, Issue 10, 2012, pp. 100908. 